EPJ Web of Conferences 7, 02002 (2010)

DOI:10.1051/epjconf/20100702002

(C) Owned by the authors, published by EDP Sciences, 2010

\title{
Heavy-ion collisions at LHC: The Alice Experiment
}

Ana Marin for the ALICE Collaboration

GSI Helmholtzzentrum für Schwerionenforschung GmbH, Germany

\begin{abstract}
We present an introduction to heavy-ion collisions at LHC with special emphasis on the ALICE experiment. The lecture is divided in three parts. The first contains a description of the new conditions for the creation of the Quark Gluon Plasma in the laboratory with HIC at the LHC in the context of the 30 years of history of HIC. The second part is a detailed explanation of the different detectors of the ALICE experiment as well as of ALICE capabilities in terms of tracking and particle identification. The last part presents a selection of the ALICE physics capabilities in soft physics, heavy quarks, quarkonia, photons and jets.
\end{abstract}

\section{References}

1. ALICE Collaboration, J. Phys. G 30, (2004) 1517

2. ALICE Coll., J. Phys. G 32, (2006) 1295

3. http://aliceinfo.cern.ch

4. R.K. Ellis, W.J. Stirling and B.R. Webber, $Q C D$ and Collider Physics.

5. R. Vogt, Ultrarelativistic Heavy-Ion Collisions.

6. P. Braun-Munzinger and J. Stachel, Nature, 448, (2007) 302

7. W.-M. et al., Journal of Physics G 33 (2006) 1.

This is an Open Access article distributed under the terms of the Creative Commons Attribution-Noncommercial License 3.0, which permits unrestricted use, distribution, and reproduction in any noncommercial medium, provided the original work is properly cited. 


\title{
Heavy lon Collisions at LHC: The ALICE experiment
}

\author{
A. Marin (GSI) \\ Dubna International Advance School of Theoretical Physics \\ Helmholtz International Summer School \\ Dense Matter in \\ Heavy Ion Collisions and Astrophysics \\ July $14^{\text {th }}-26^{\text {th }} 2008$, Dubna (Russia)
}

\section{Table of Contents}

- Introduction to Heavy Ion Collisions

- The LHC

- The ALICE experiment

- ALICE physics:

- Soft Physics

- Heavy quarks and quarkonia

- Photons, Jets and high $p_{T}$ 


\section{Goals of High Energy Heavy-Ion Collisions}

- Understand 2 basic properties of the strong interaction: (de)confinement, chiral symm. breaking/restoration

- Probe conditions quark-hadron phase transition in primordial Universe (few $\mu \mathrm{sec}$ after the Big Bang)

- Study the phase diagram of QCD matter: produce and study the QGP

- Study the regime of non-linear (high density) many-body parton dynamics at small-x (CGC).

\section{Quantum Chromodynamics (QCD)}

-QCD is a gauge field theory that describes the strong interaction of colored quarks and gluons

-QCD potential

$$
V=-\frac{4}{3} \frac{\alpha_{s}}{r}+k r
$$

- Asymptotic freedom at short distance - Confinement at large distance

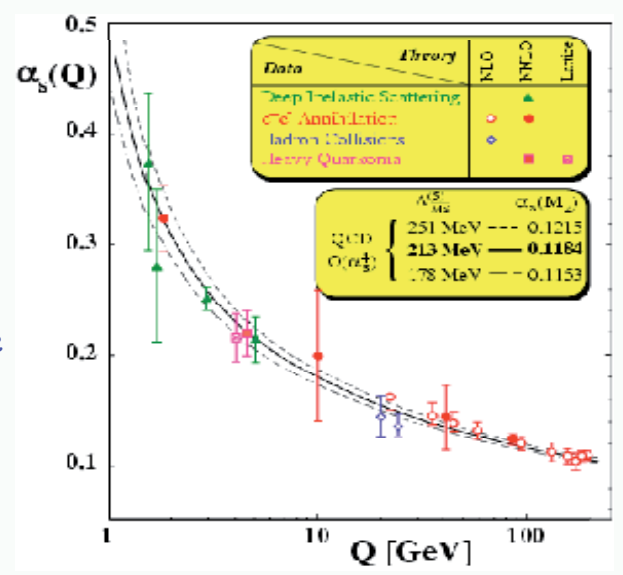

S Bethke, J. Phys. G26 (2000) R27 
Asymptotic Freedom

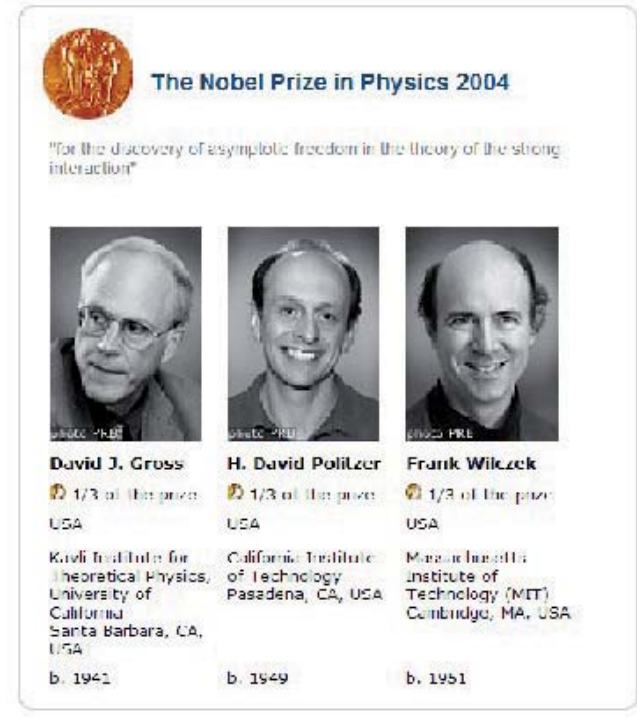

"What this year's Laureates discovered was something that, at first sight, seemed completely contradictory. The interpretation of their mathematical result was that the closer the quarks are to each other, the weaker is the 'colour charge'. When the quarks are really close to each other, the force is so weak that they behave almost as free particles. This phenomenon is called 'asymptotic freedom'. The converse is true when the quarks move apart: the force becomes stronger when the distance increases."

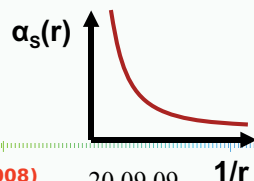

\section{Lattice QCD: deconfinement and chiral} symmetry restoration

Lattice provides a first-principles approach to studies of large distances, non-perturbative aspects of QCD
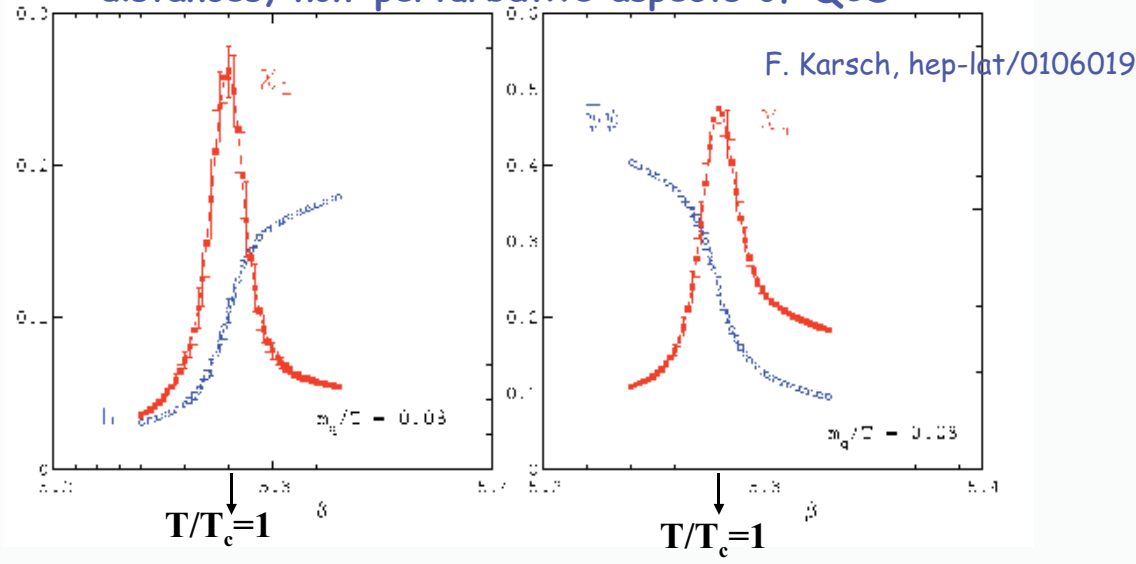

$\chi_{L}:$ Polyakov loop susceptibility

$\chi_{m}:$ chiral susceptibility

\section{The maxima of $\chi_{L}$ and $\chi_{m}$ coincide}




\section{Lattice QCD: Pressure and energy density}

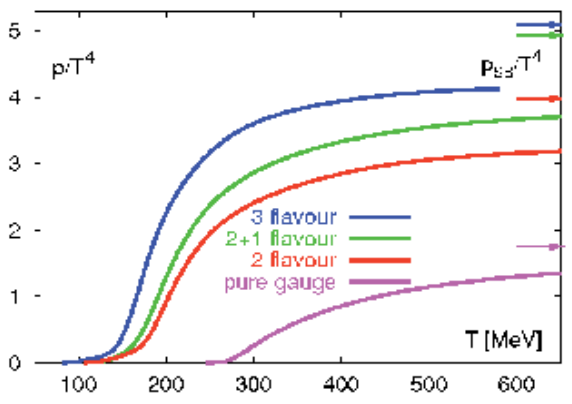

F. Karsch, hep-lat/0106019

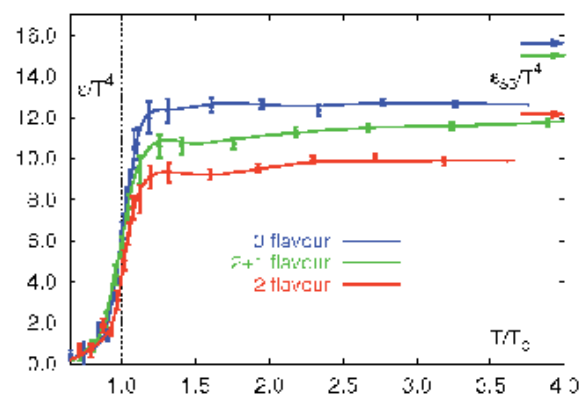

Transition to QGP: $T_{c}=175 \pm 15 \mathrm{MeV}: \varepsilon_{c}=0.7 \pm 0.2 \mathrm{GeV} / \mathrm{fm}^{3}$

Above $T_{c}$ the energy goes into

more degrees of freedom

M. Cheng et al., Phys. Rev. D77 (2008) 014511

ense Matter in Heavy Ion Collisions and Astrophysics, A. Marin (Dubna July 2008) $\quad 20.09 .09$

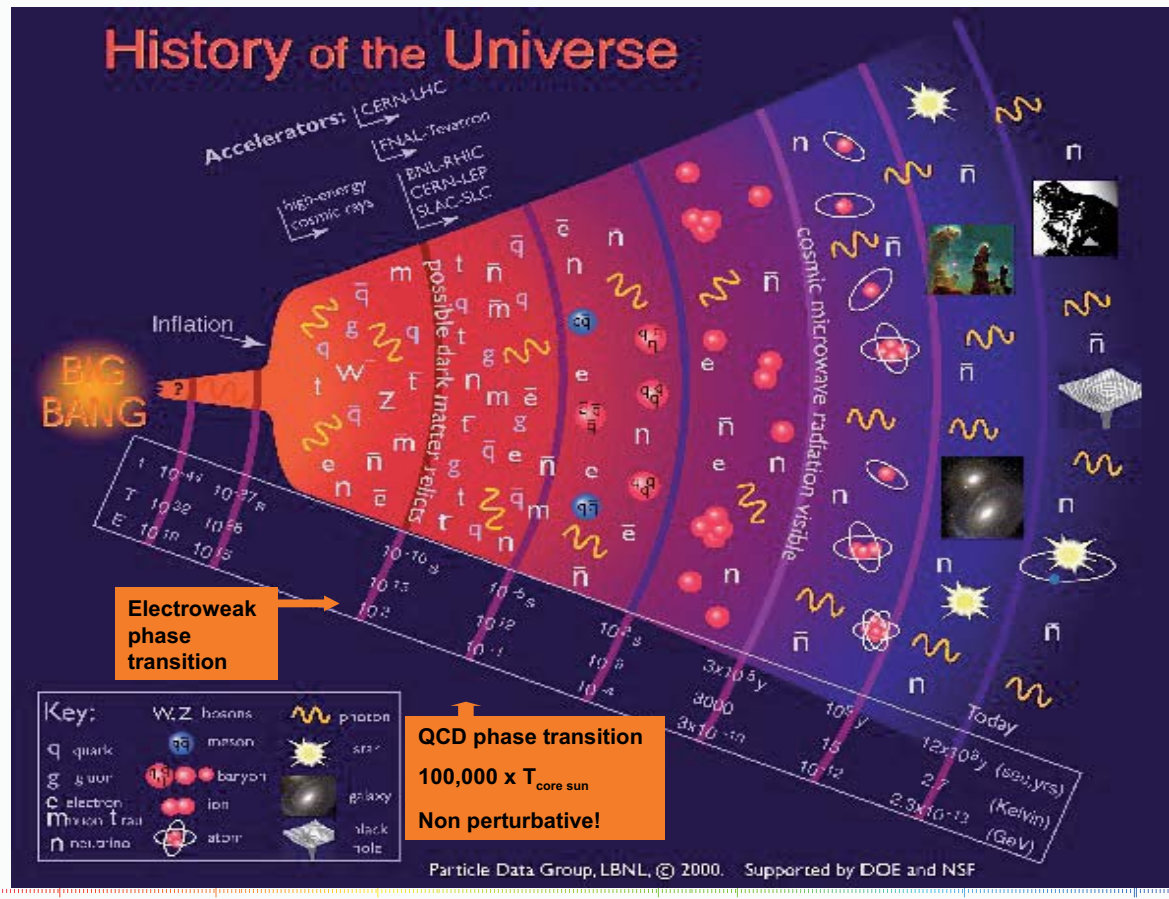

ense Matter in Heavy Ion Collisions and Astrophysics, A. Marin (Dubna July 2008)

20.09 .09 


\section{QGP in the laboratory}

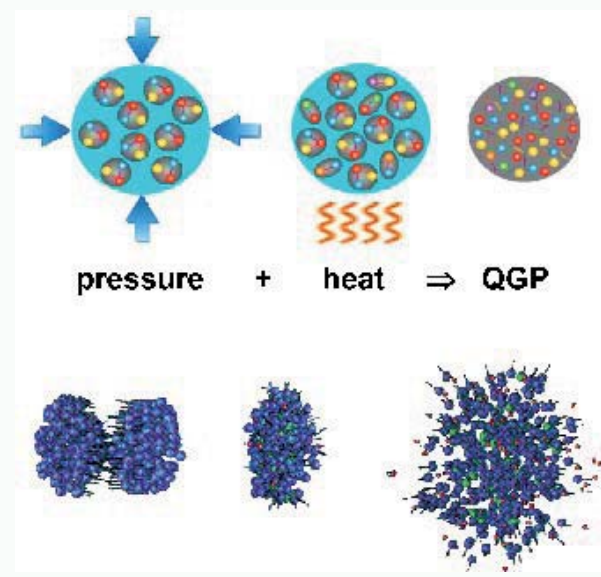

Key parameters: Bombarding energy and collision centrality

\section{Schematic space-time evolution of a HIC}

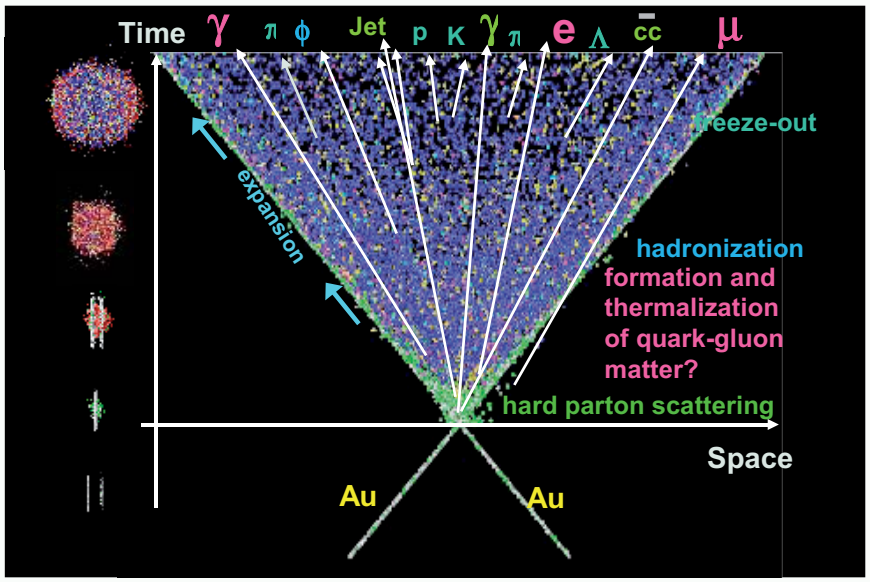

Different observables sensitive to different reaction stages 


\section{History of Heavy Ion Collisions}

- Bevalac (LBL)

- fixed target (1975-1986) $\sqrt{s}<2.4 \mathrm{GeV}$

- SIS (GSI)

- fixed target (1989-) $\sqrt{s}<2.7 \mathrm{GeV}$

- $A G S$ (BNL)

- fixed target (1986-1998) $\sqrt{s}<5 \mathrm{GeV}$

- SPS (CERN)

- fixed target (1986-2003) $\sqrt{s}<20 \mathrm{GeV}$

- RHIC (BNL)

- collider (2000-) $\sqrt{s}<200 \mathrm{GeV}$

- LHC (CERN)

- collider (2008-) $\sqrt{s}<5500 \mathrm{GeV}$

- faIR (GSI)

- fixed target (2014-) $\sqrt{s}<9 \mathrm{GeV}$

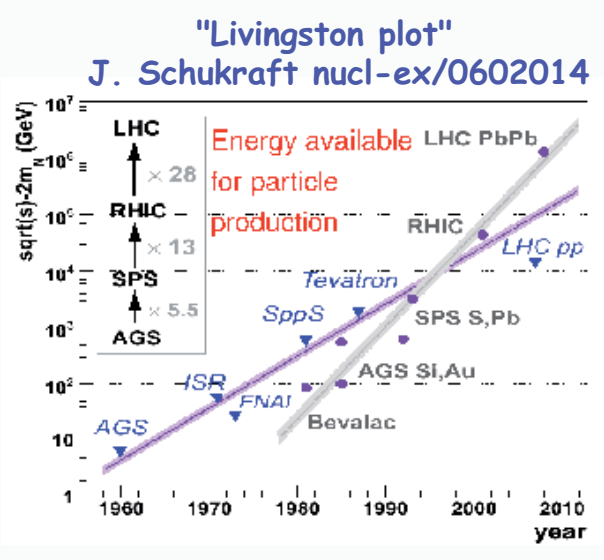

Energy doubling every 4 (1.7) years for $p$ (ion) beams.

\section{Phase diagram of matter}

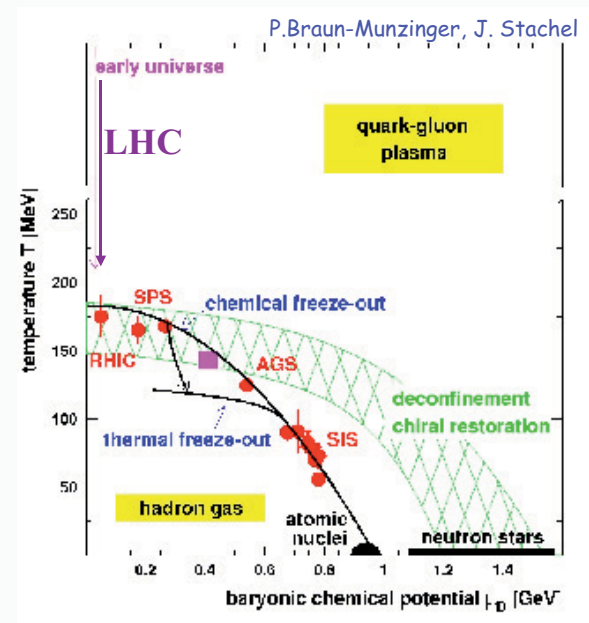

Transition from hadronic to quark matter

Chiral symmetry restoration 


\section{Energy density: Bjorken estimate}
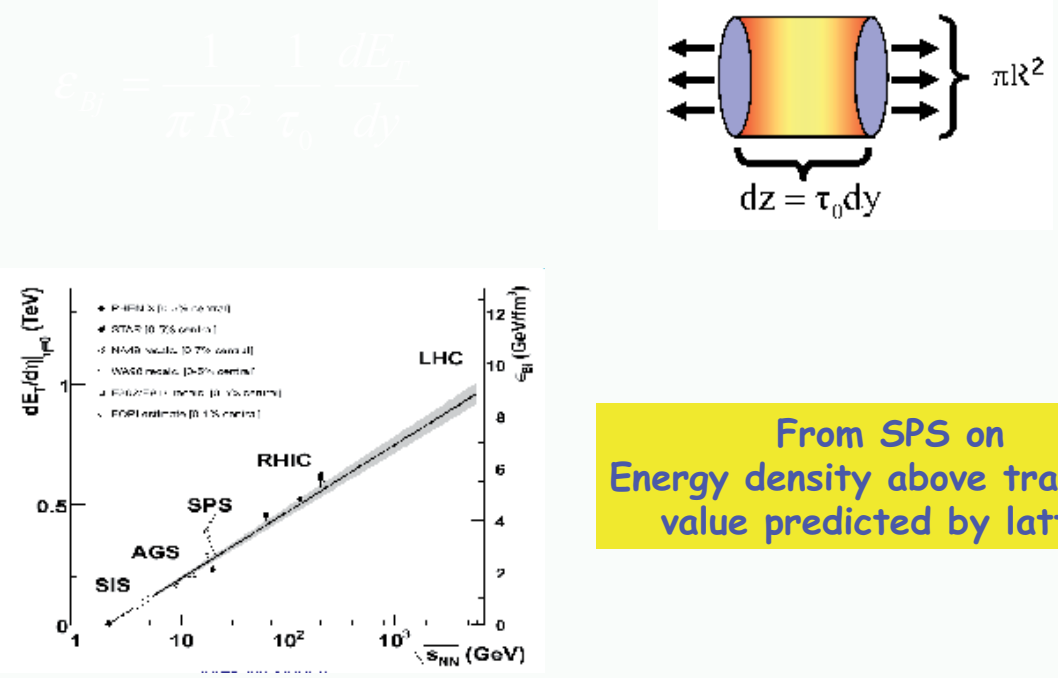

From SPS on

Energy density above transition value predicted by lattice

\section{THE LHC}




\section{The CERN accelerator complex}

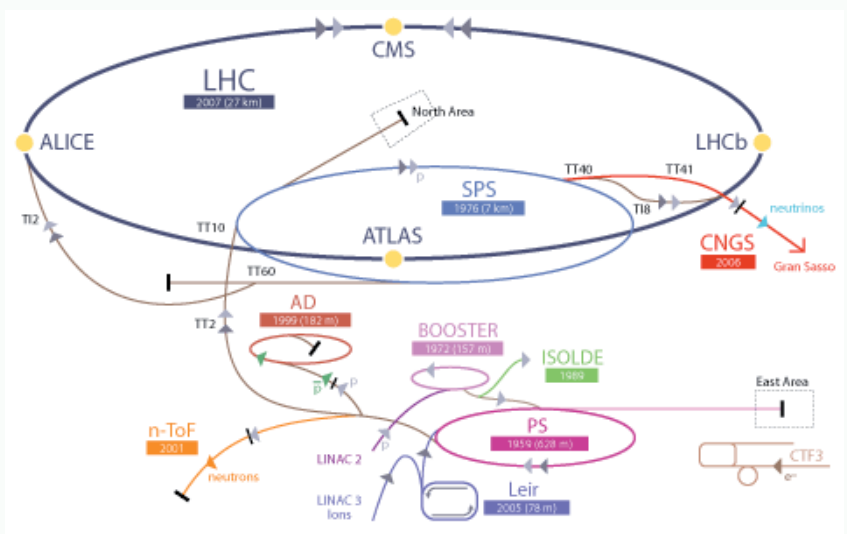

LINAC2- BOOSTER-PS-SPS-LHC

\section{The Large Hadron Collider \\ Hadron Collider}

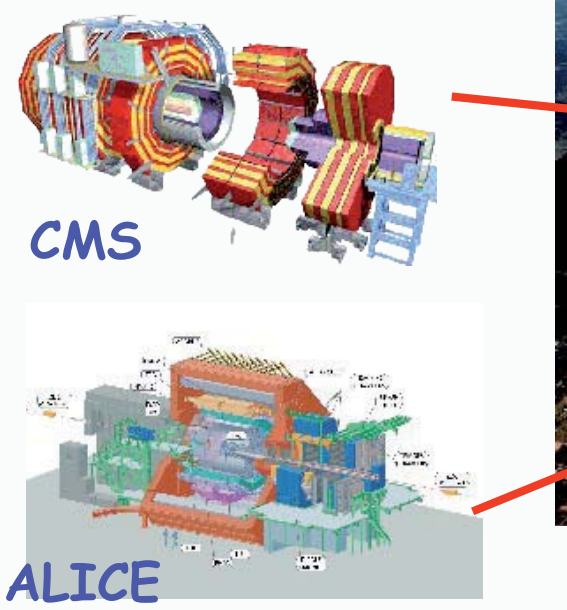

p+p@ $14 \mathrm{TeV}$

$\mathrm{Pb}+\mathrm{Pb} @ 5.5 \mathrm{~A} \mathrm{TeV}$
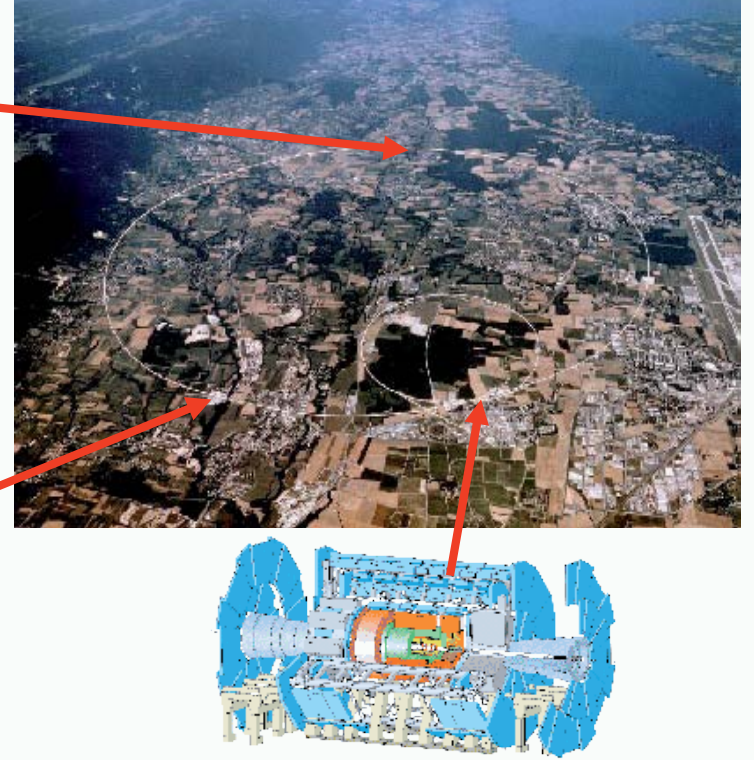

ATLAS 


\section{Physics questions at LHC}

- ATLAS, CMS, LHCb:

- Higss mechanism

- Supersymmetry

- CP violation

- ALICE:

- Chiral Symmetry breaking

- Origin of mass of hadrons

- Deconfinement

- Hadronization

- ALL:

- Understand high energy nuclear interactions (for cosmic ray studies)

\section{Heavy ion collisions at LHC}

\begin{tabular}{|c|c|c|c|}
\hline & SPS & RHIC & LHC \\
\hline${\sqrt{\mathrm{S}_{\mathrm{NN}}}}(\mathrm{GeV})$ & 17 & 200 & 5500 \\
\hline $\mathrm{dN}_{\mathrm{ch}} / \mathrm{dy}$ & 430 & 730 & $1000-4000$ \\
\hline$\tau_{{ }_{\mathrm{QGP}}(\mathrm{fm} / \mathrm{c})}$ & 1 & 0.2 & 0.1 \\
\hline $\mathrm{T}_{\mathrm{T}}$ & 1.1 & 1.9 & $3.0-4.7$ \\
\hline$\left.\varepsilon \mathrm{GeV}_{\mathrm{c}}(\mathrm{Gm})^{3}\right)$ & 3 & 5 & $15-60$ \\
\hline$\tau_{\mathrm{QGP}}(\mathrm{fm} / \mathrm{c})$ & $\leq 2$ & $2-4$ & $\geq 10$ \\
\hline$\tau_{\mathrm{f}}(\mathrm{fm} / \mathrm{c})$ & $\sim 10$ & $20-30$ & $15-60$ \\
\hline $\mathrm{V}_{\mathrm{f}}\left(\mathrm{fm}^{3}\right)$ & few $10^{3}$ & few $10^{4}$ & few $10^{5}$ \\
\hline
\end{tabular}

faster hotter denser longer

bigger 


\section{Heavy Ion collisions at LHC}

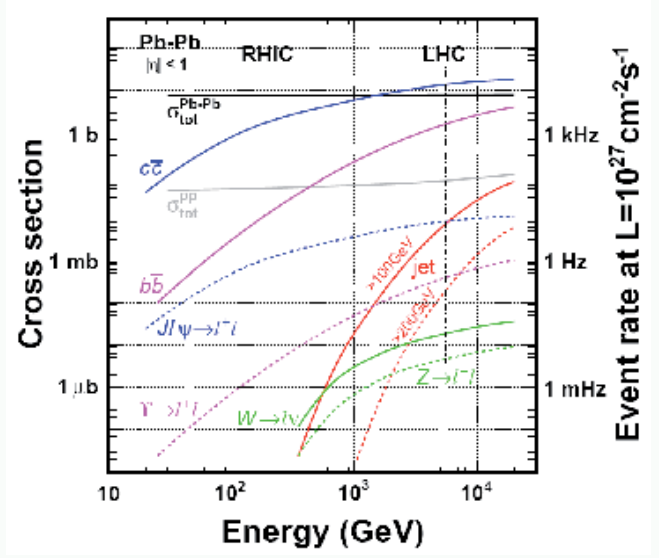

Cross-sections of interesting probes expected to increase by factors

$$
\begin{aligned}
& \sim 10 \text { ( } \mathrm{cc} \text { ) to } \\
& \sim 10^{2} \text { ( bb) to }
\end{aligned}
$$

$\sim>10^{5}$ (very high $p_{T}$ jets) : Hard probes of the medium accessible at LHC

Direct photons are abundantly produced at LHC

\section{Hard processes at LHC}

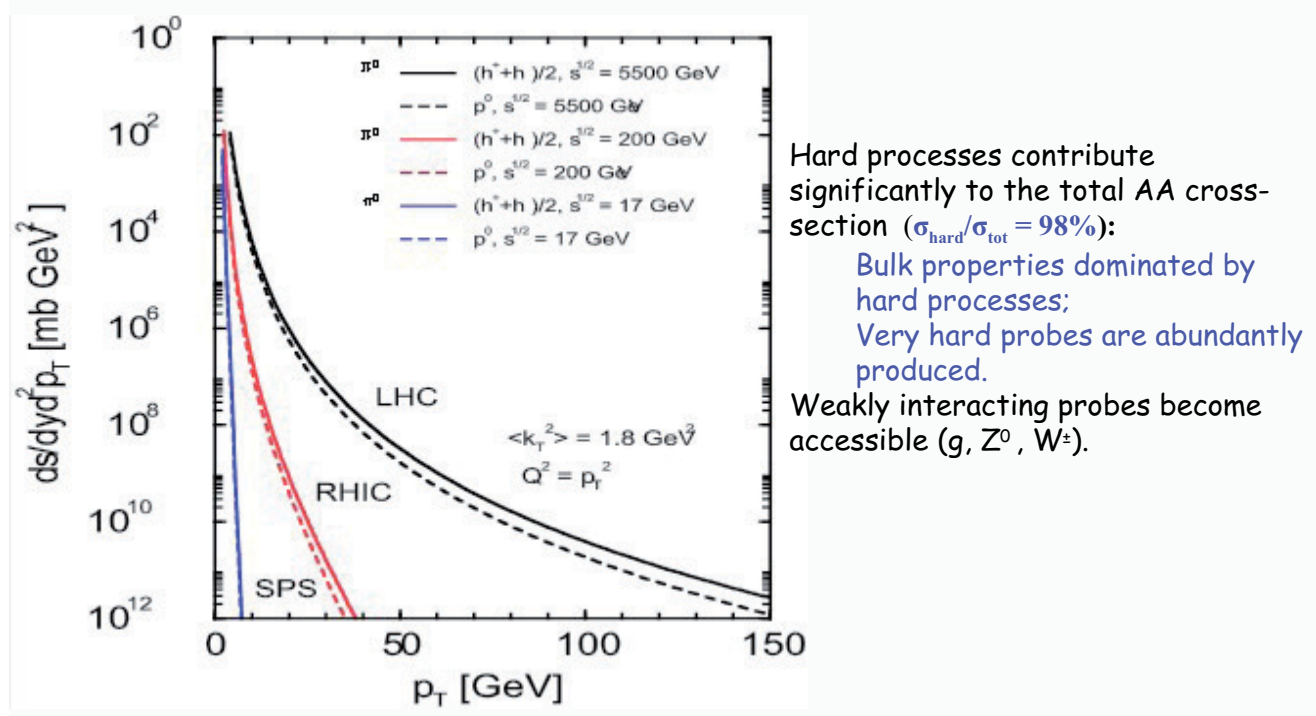

K. Kajantie, Nucl Phys. A 715(2003) 432 


\section{Physics topics of ALICE}

- Event characterization Multiplicity, centrality

- Bulk properties of the hot and dense medium (dynamics of hadronization) Hadron ratios, hadronic resonances

- Chiral symmetry restoration Short lived resonance masses

- Space-time fireball description and fireball expansion dynamics Momentum correlations (HBT), Radial and anisotropic flow

- Heavy quark production Charmonium and bottonium (Debye screening, recombination(?))

- Partonic energy loss in QGP Jet reconstruction, Jet quenching, high pt spectra, inclusive high p.suppression, open charm (Mass/color dependence of $E$ loss)

- Fluctuation phenomena Event-by-Event analysis

\section{ALICE: The dedicated HI Experiment}

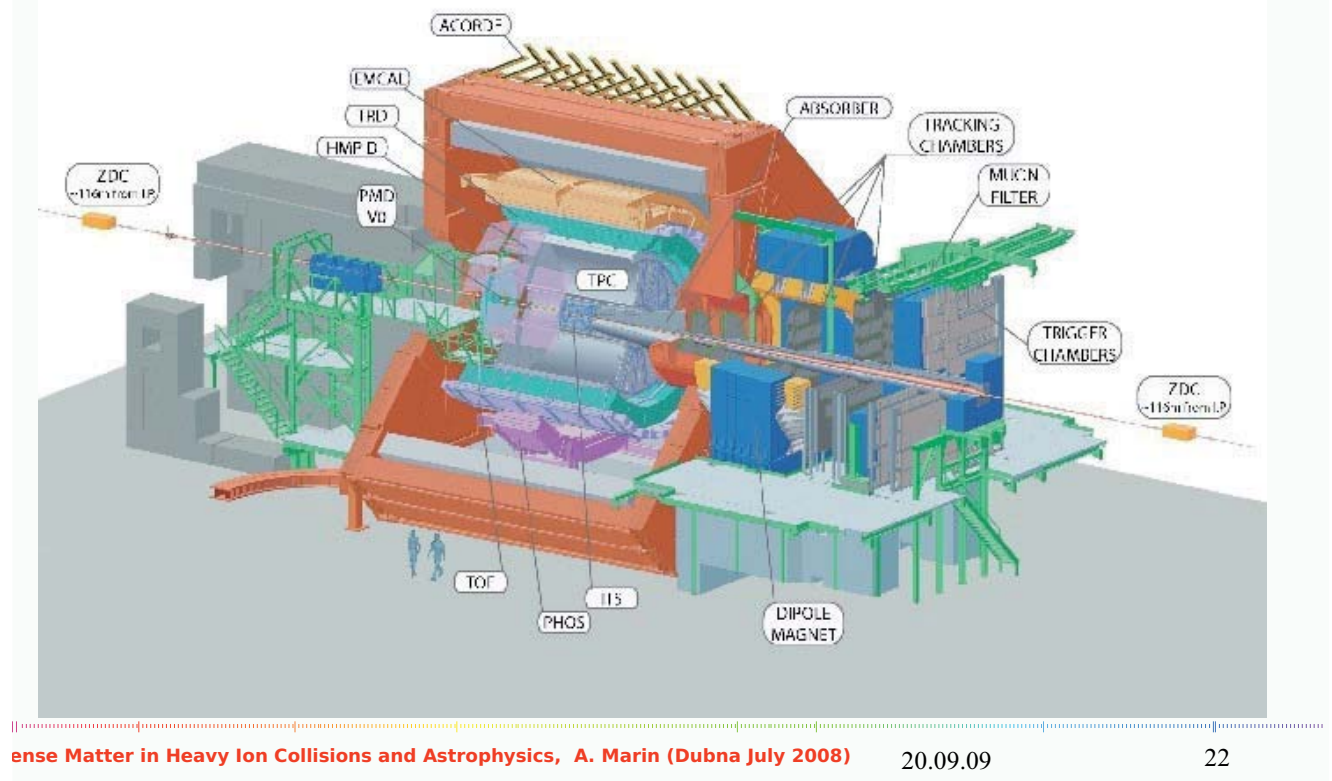




\section{ALICE Collaboration}
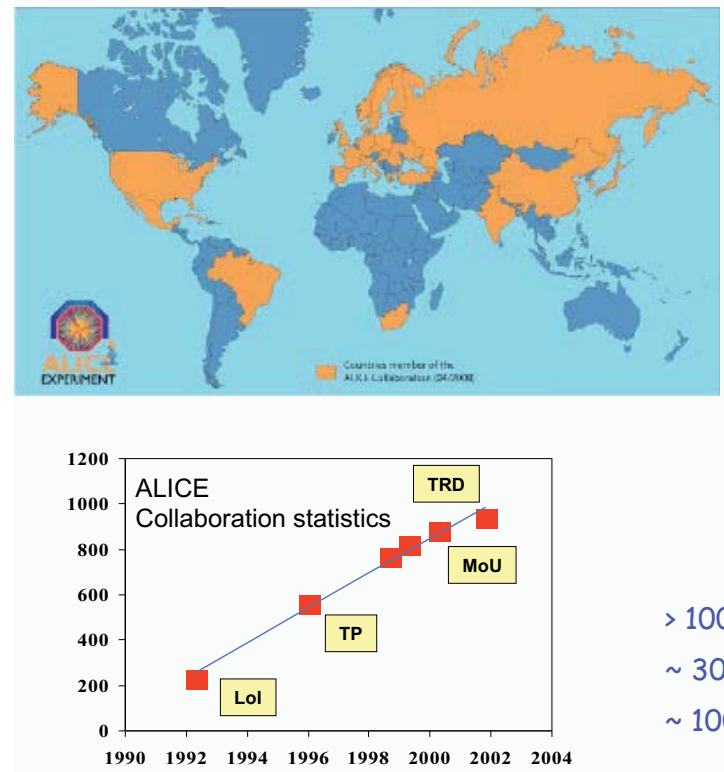

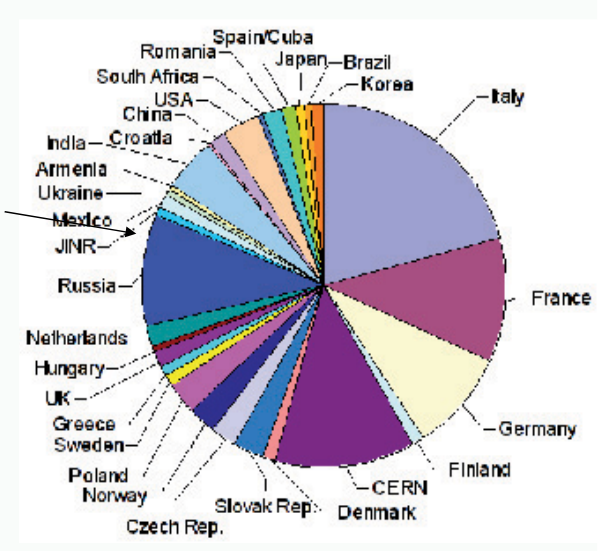

$>1000$ Members

$\sim 30 \quad$ Countries

$\sim 100$ Institutes

\section{The Inner Tracking System}

\section{Layers of silicon detectors for precision tracking in $|\eta|<0.9$}

- Three technologies to keep occupancy $\sim 2 \%$ from $R_{\min } \sim 4 \mathrm{~cm}\left(80\right.$ tracks $\left./ \mathrm{cm}^{2}\right)$

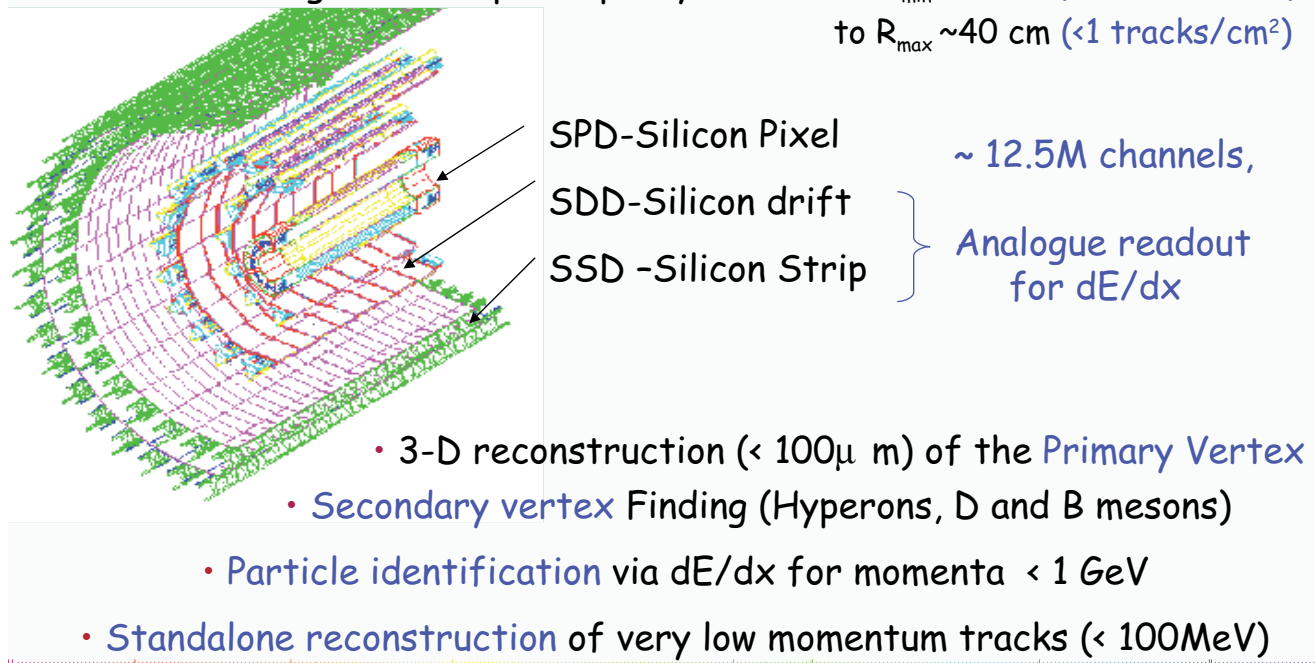




\section{The ITS assembly}
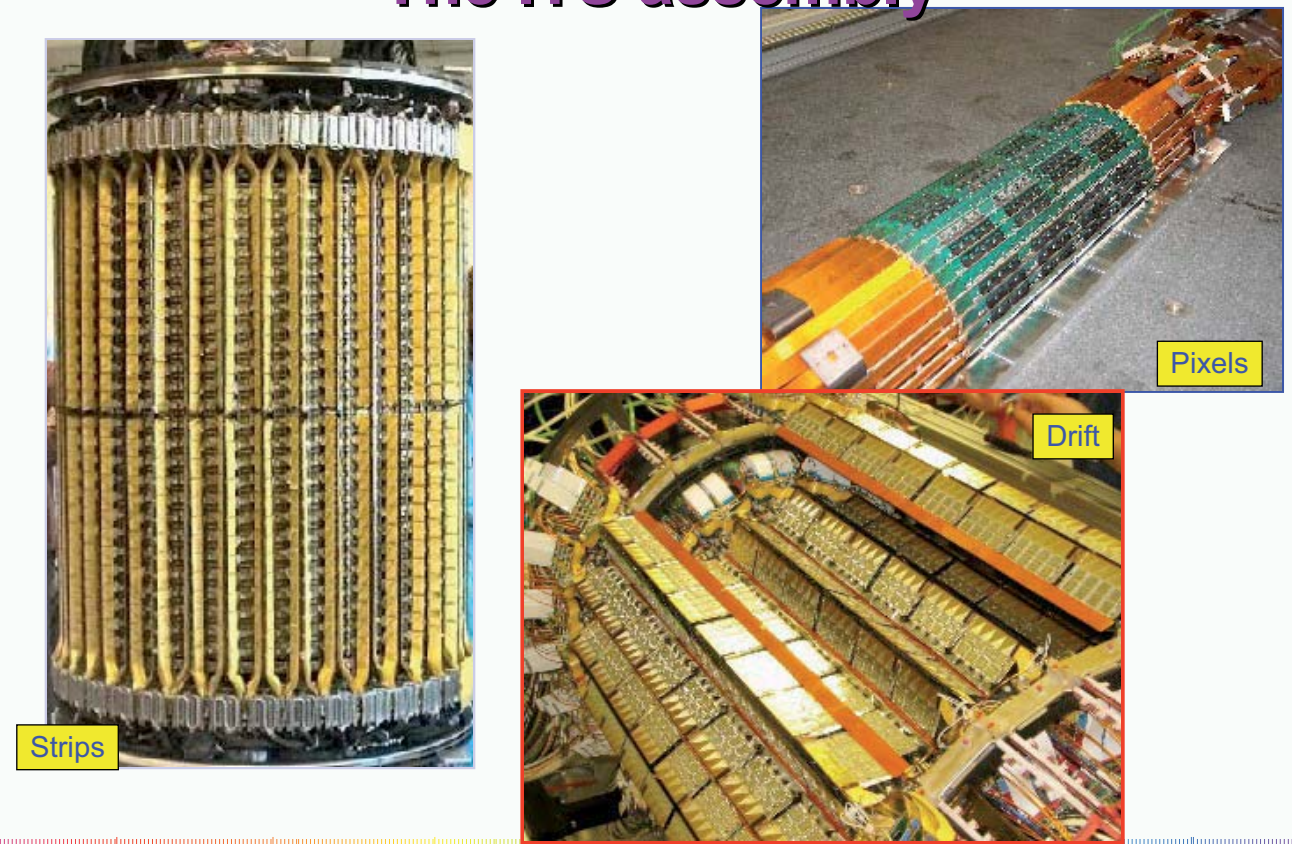

20.09.09

25

\section{The Time Projection Chamber}

\section{Main tracking detector (charged particles) of the ALICE Central Barrel}

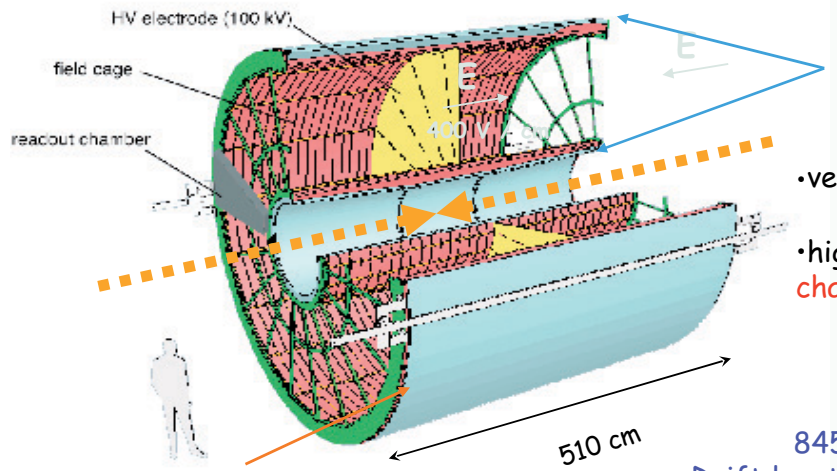

Inner and Outer

Containment Vessels

$\left(150 \mathrm{~mm}, \mathrm{CO}_{2}\right)$

-very thin \& lightweight FC 3\% $X_{0}$

-high track density \& small space

charge

drift gas, chamber lay-out, field strips, ..

$845<r<2466 \mathrm{~mm}$

Suspended field

Drift length $2 \times 2500 \mathrm{~mm}$

defining strips

Drift gas $\mathrm{Ne}-\mathrm{CO}_{2}-\mathrm{N}_{2}(86 / 9 / 5)$

$400 \mathrm{~V} / \mathrm{cm}$

Gas volume $95 \mathrm{~m} 3$

557568 readout pads 


\section{The TPC}

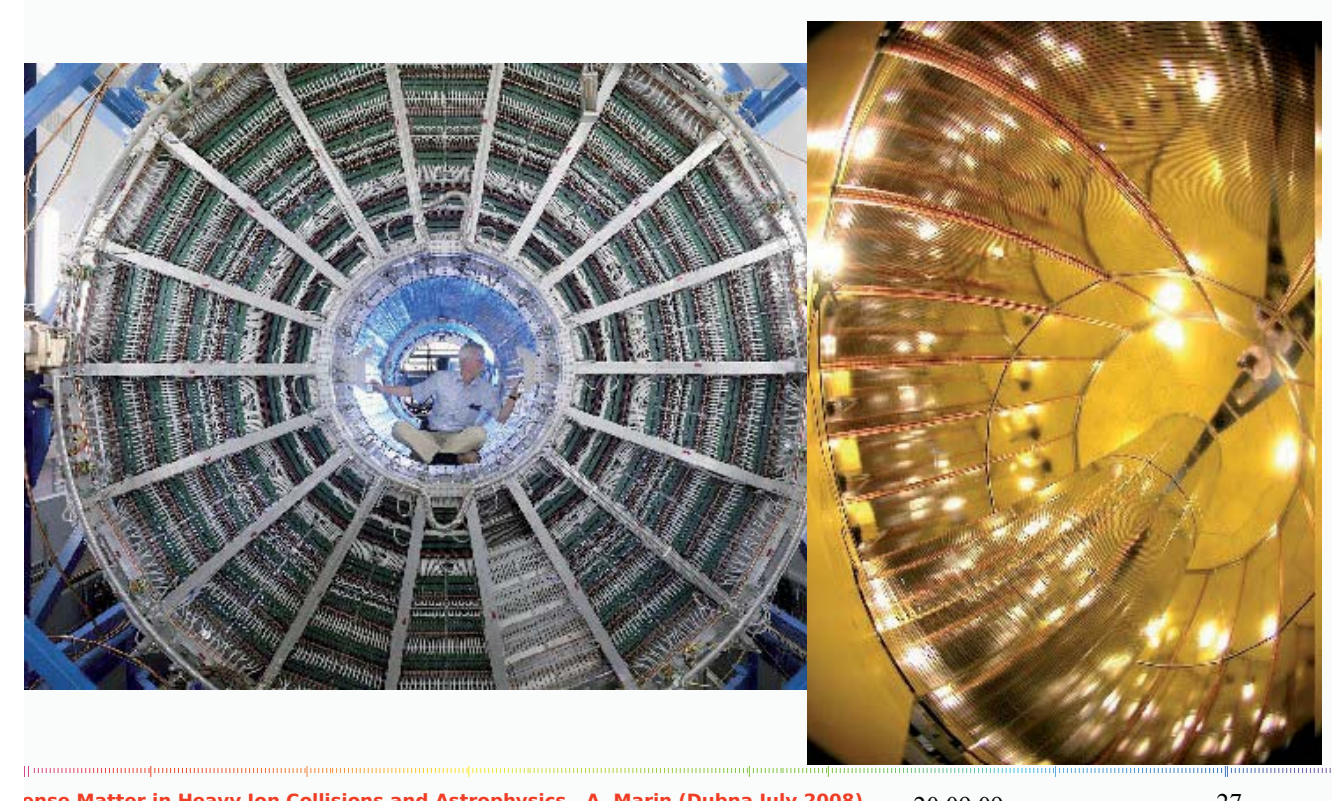

ense Matter in Heavy lon Collisions and Astrophysics, A. Marin (Dubna July 2008) 20.09.09

27

\section{The TPC installation}

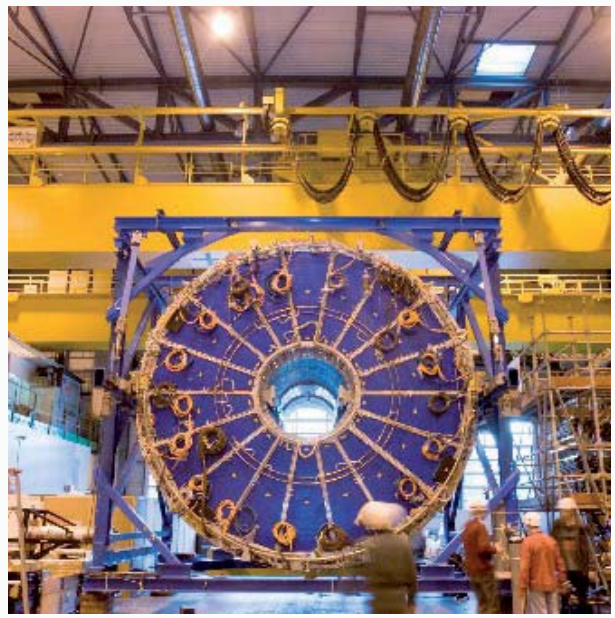

\section{January 2007}

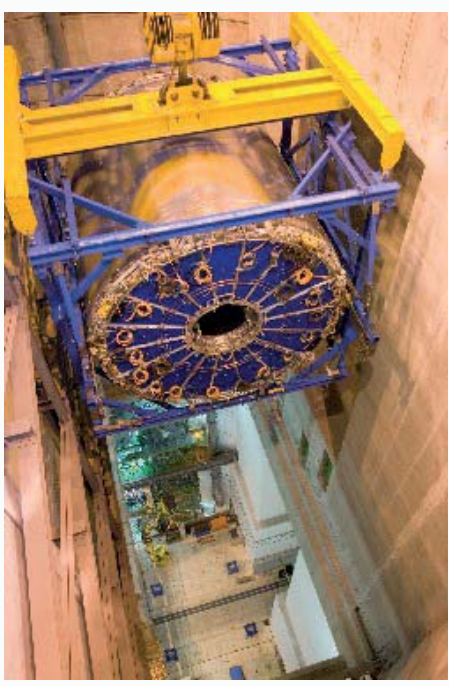

20.09.09

28 


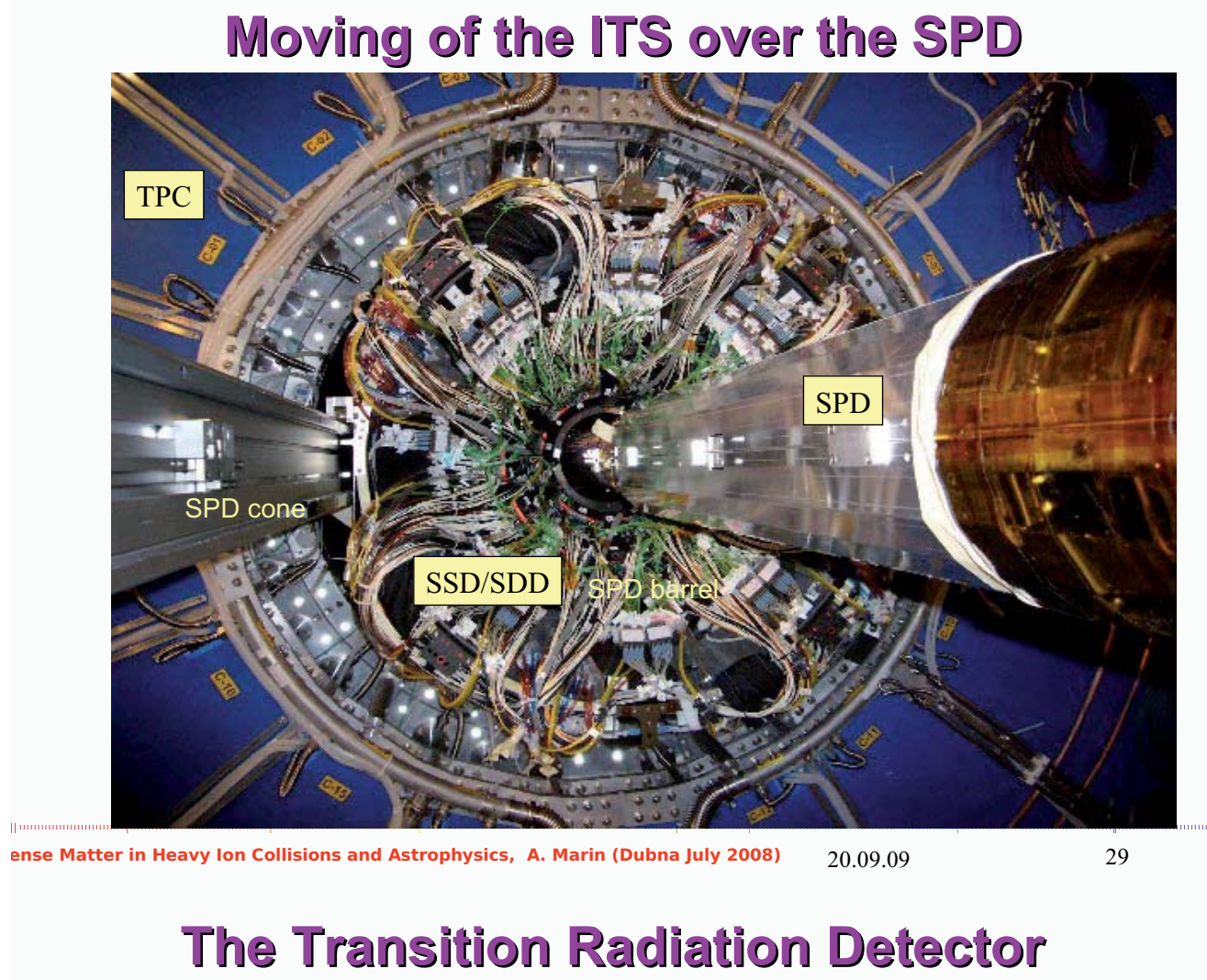

e-identification

- 18 supermodules

- 6 radial layers

- 5 longitudinal stacks

$\Rightarrow 540$ chambers

$\Rightarrow 750 \mathrm{~m}^{2}$ active area

$\Rightarrow 28 \mathrm{~m}^{3}$ of gas

Each chamber:

$\approx 1.45 \times 1.20 \mathrm{~m}^{2}$

$\approx 12 \mathrm{~cm}$ thick (incl.Radiators and electronics)

in total 1.18 million read out channels

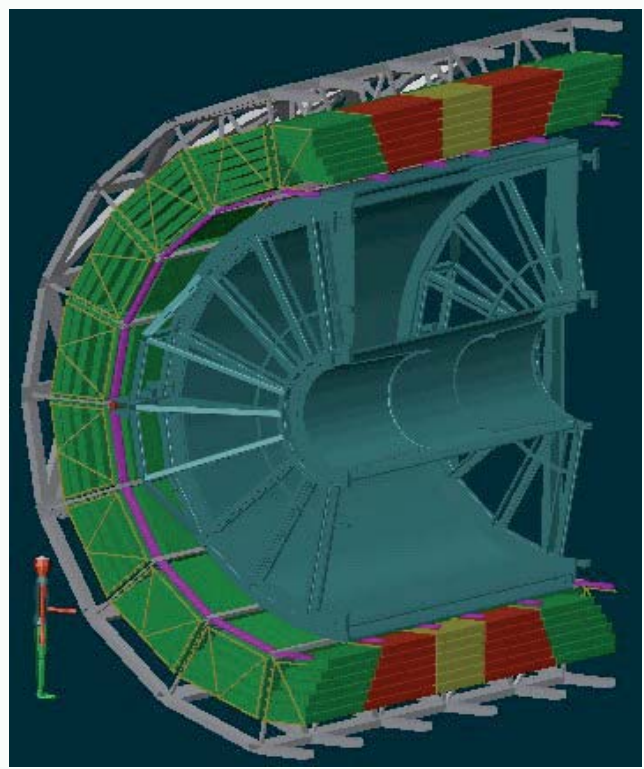




\section{ALICE TRD principle}
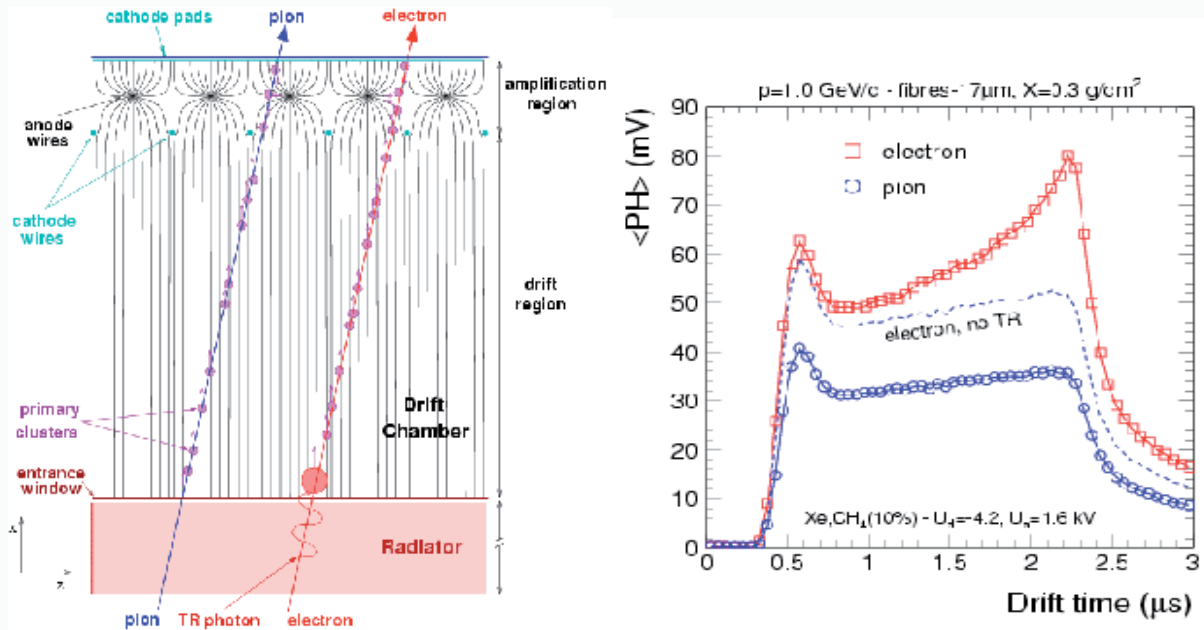

Charged particles at $\gamma>1000$ give T.R. photons ( $<30 \mathrm{KeV})$.

They are absorbed by high- $\mathrm{Z}$ gas $\left(\mathrm{Xe}, \mathrm{CO}_{2}\right)$ mixture

ense Matter in Heavy lon Collisions and Astrophysics, A. Marin (Dubna July 2008) 20.09.09

\section{TRD assembly and installation}
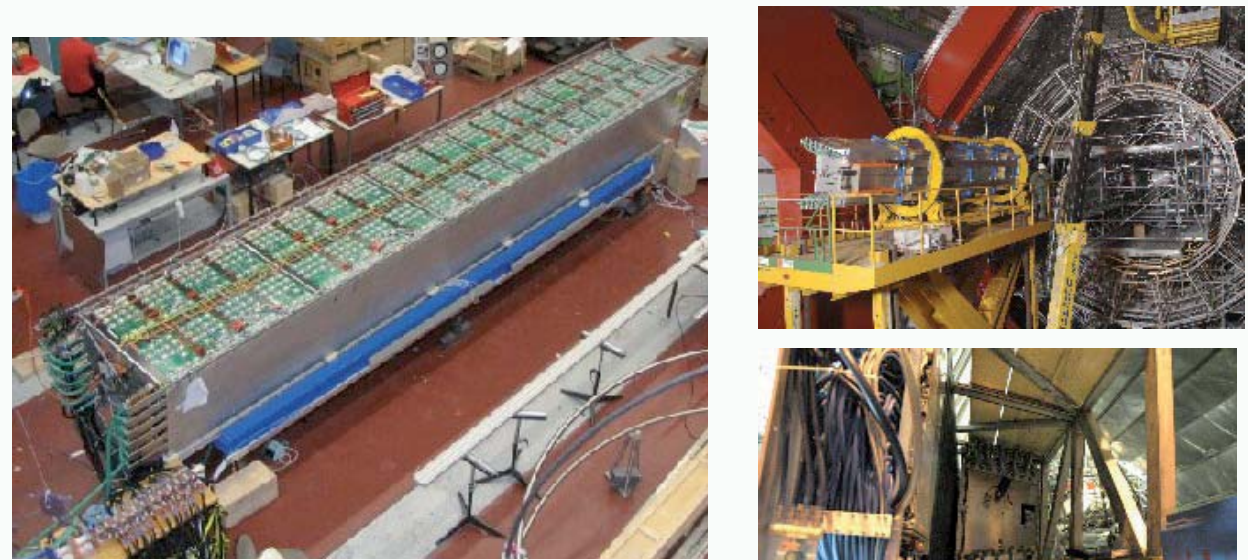

\section{SM's are installed}

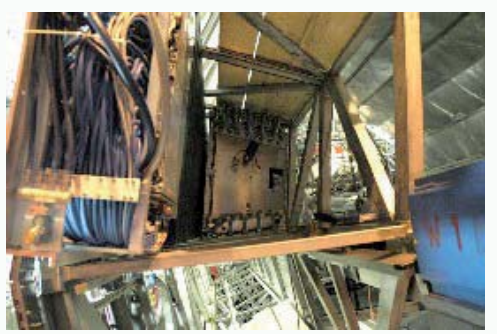




\section{The Time Of Flight System}

Large array at $R \sim 3.7 \mathrm{~m}$, covering $|\eta|<0.9$ and full $\phi$, requirements:

- Time resolution < 100 ps

- Very high granularity, $O\left(10^{5}\right)$ channels to keep occupancy $<15 \%$

Active surface $150 \mathrm{~m}^{2}$

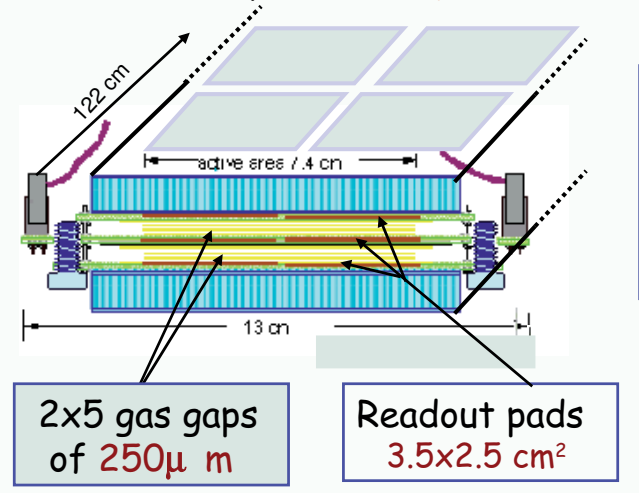

TOF basic element: double-stack Multigap RPC strip

$7.4 \times 120 \mathrm{~cm}^{2}$ active area segmented into 96 readout pads

Extensive R\&D, from TB data:

-Intrinsic Resolution 40 ps - Efficiency $>99 \%$

Full TOF: 1638 strips, arranged in $18 \phi$ sectors, each of 5 modules along $z$

\section{TOF supermodule}

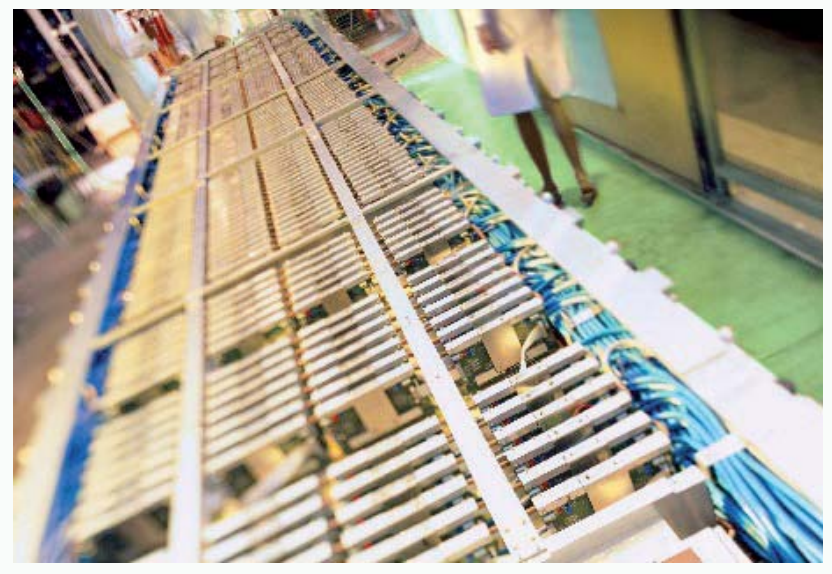




\section{The High Momentum Particle ID Detector}

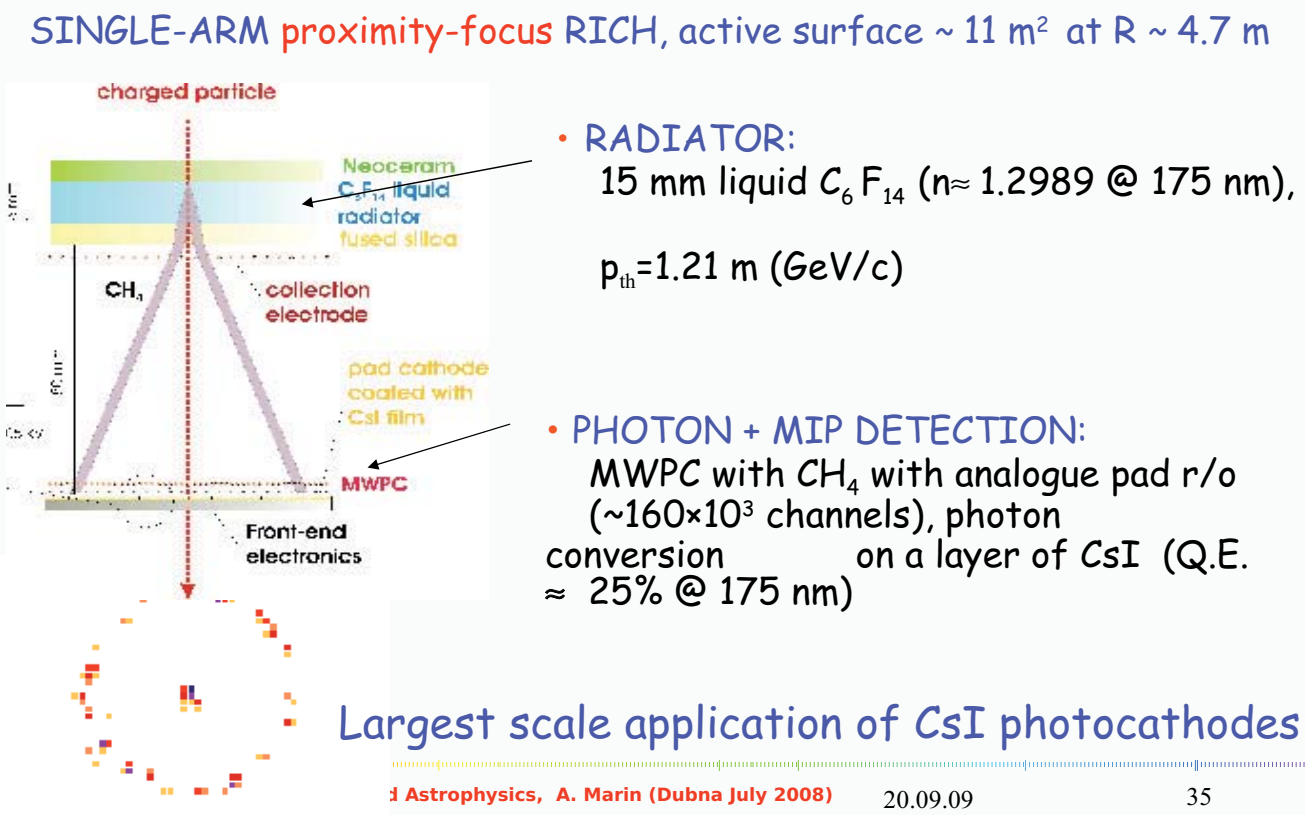

\section{The HMPID detector during installation}

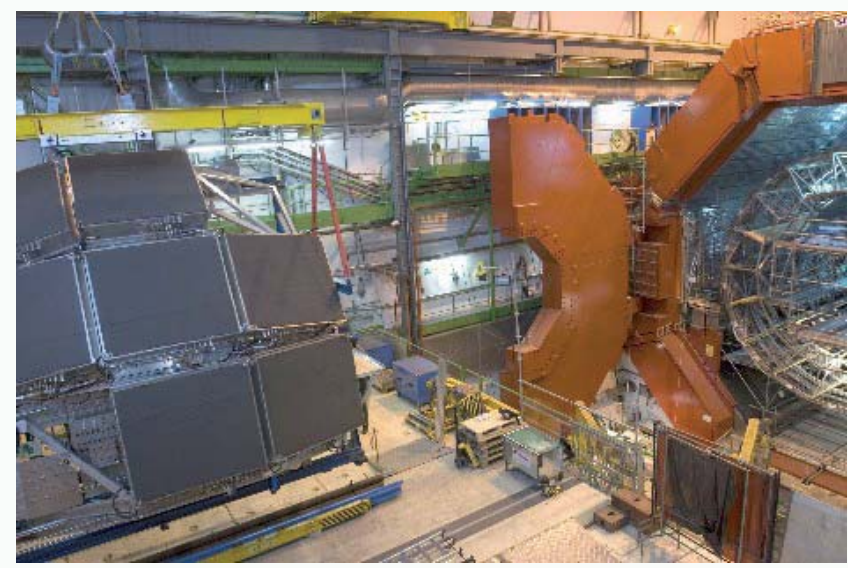




\section{PHOTON SPECTROMETER}

- Dense like lead and transparent like crystal to stop photons

- $\mathrm{PbWO}_{4}$ crystal (17920 cristals in total)

- $R_{\| l}=2.2 \mathrm{~cm}, X_{0}=8.9 \mathrm{~mm}, \rho=8.28 \mathrm{~g} / \mathrm{cm}^{3}$,

- $\mathrm{n}=2.16$, size: $22 \times 22 \times 180 \mathrm{~mm}^{3}$

PHOS has 5 modules installed at $4.6 \mathrm{~m}$ apart from the ALICE interaction point.
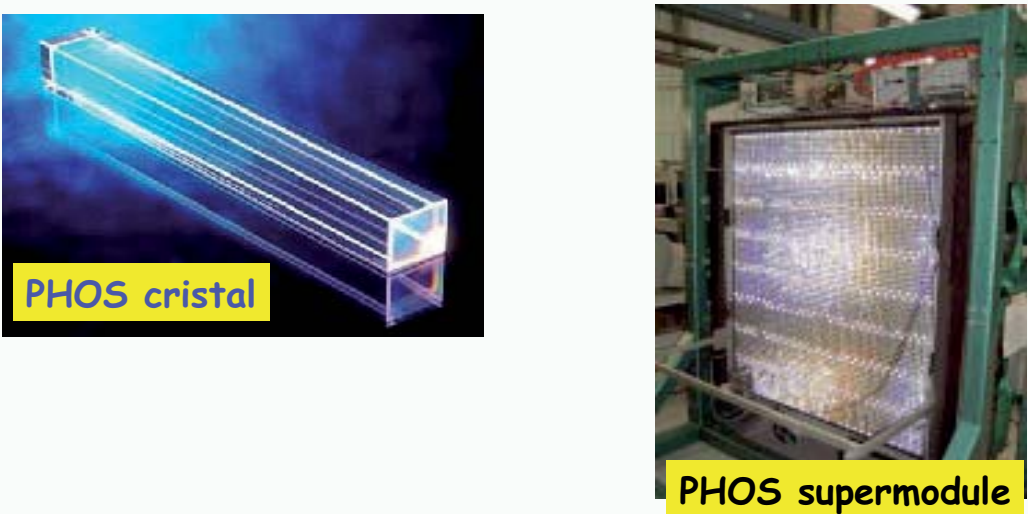

\section{The Muon arm}

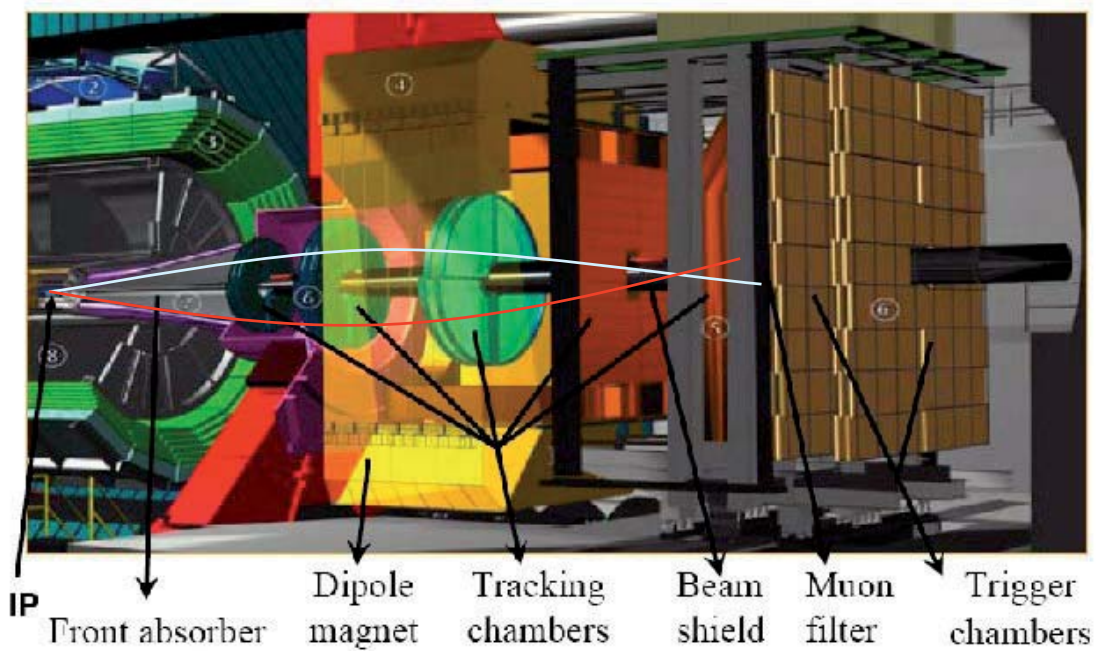

Muon spectrometer is dedicated to the (di-)muons measurement $(2.5<\eta<4)$ 


\section{Muon arm assembly}

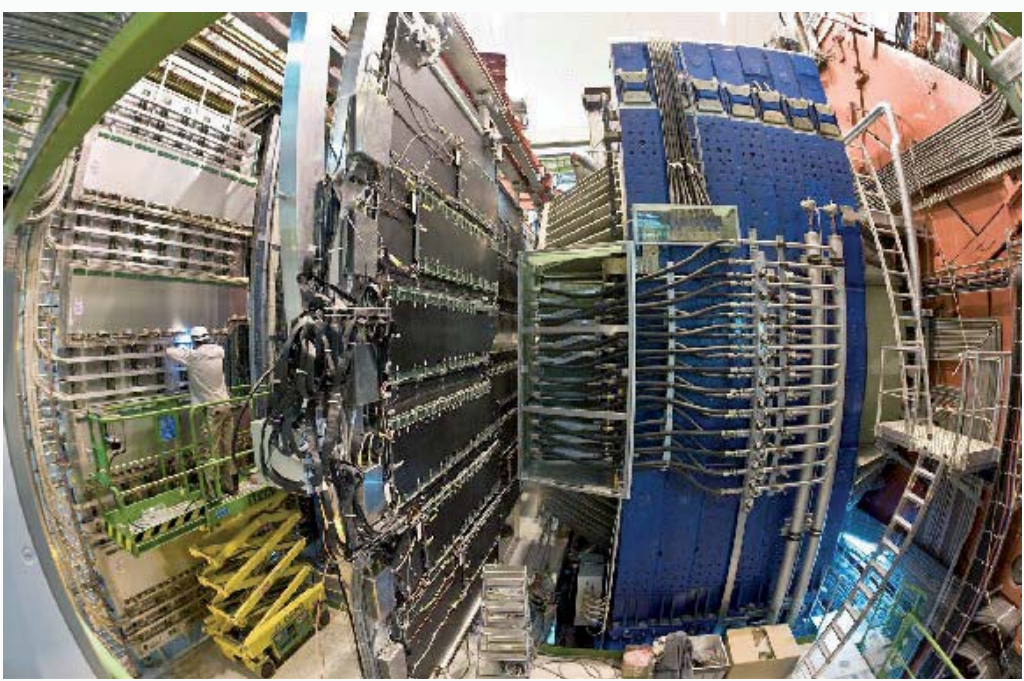

\section{Forward Detectors}

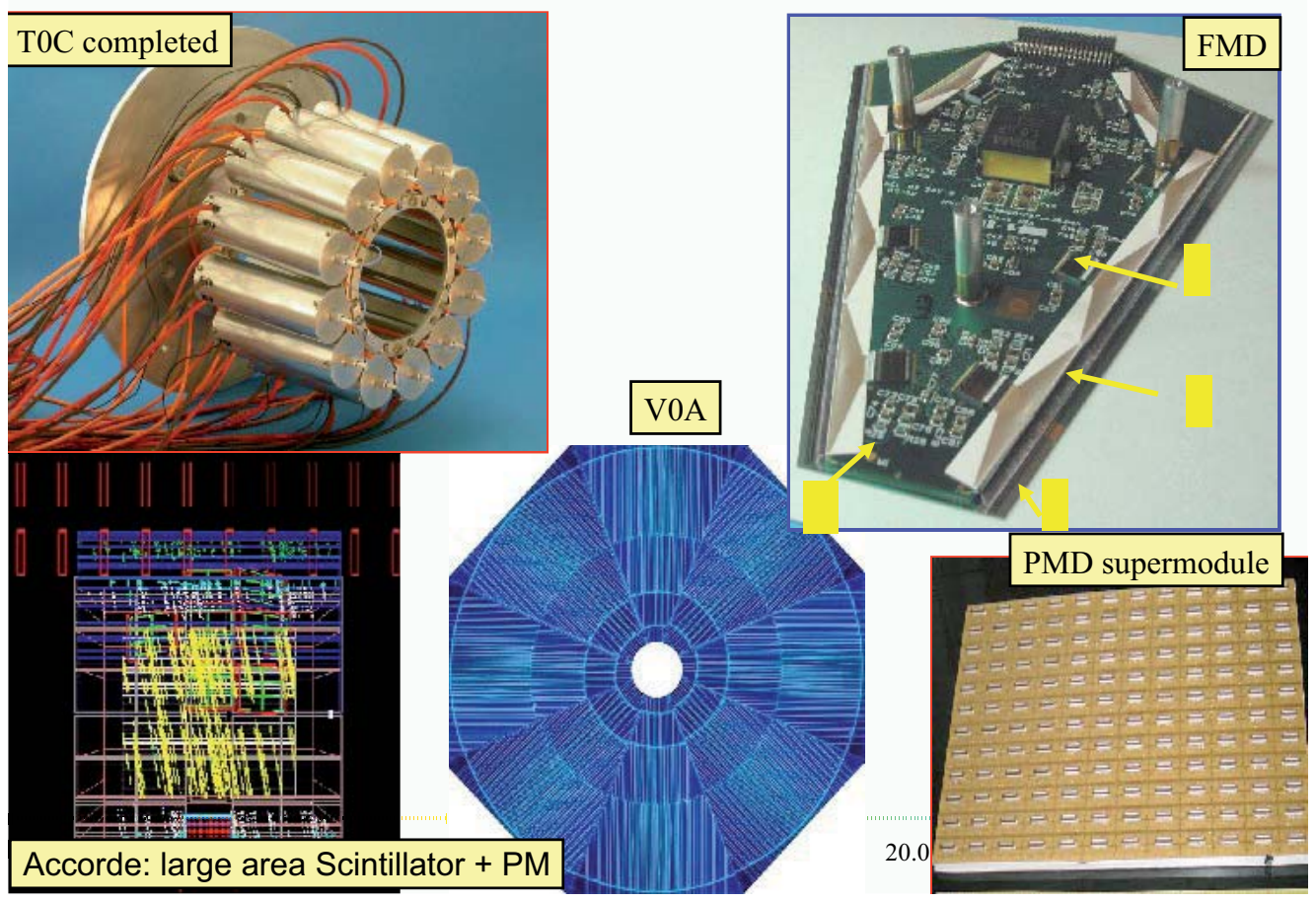




\section{Zero Degree Calorimeter}

- 6 small \& dense calorimeters

- $8 \mathrm{~m}$ (em calo) and $116 \mathrm{~m}$ (ZP, ZN) from IP

- trigger on impact parameter (spectators)

- $v_{1}$ measurement

- unbiased event plane

\begin{tabular}{|c|c|c|c|}
\cline { 2 - 4 } \multicolumn{1}{c|}{} & $\begin{array}{c}\text { Proton } \\
\text { ZDC (ZP) }\end{array}$ & $\begin{array}{c}\text { Neutron } \\
\text { ZDC (ZN) }\end{array}$ & $\begin{array}{c}\text { EM } \\
\text { ZDC }\end{array}$ \\
\hline $\begin{array}{c}\text { Dimensions } \\
\left(\mathrm{cm}^{3}\right)\end{array}$ & $12 \times 21 \times 150$ & $7 \times 7 \times 100$ & $7 \times 7 \times 21$ \\
\hline Absorber & brass & W-alloy & lead \\
\hline $\begin{array}{c}\text { Fibre angle } \\
\text { wrt LHC axis }\end{array}$ & $0^{\circ}$ & $0^{\circ}$ & $45^{\circ}$ \\
\hline Fibre $\varnothing(\mu \mathrm{m})$ & 550 & 365 & 550 \\
\hline
\end{tabular}

- impact parameter also in pA ('grey \& black' tracks)

- effective $\mathrm{A}^{\alpha}$ dependences
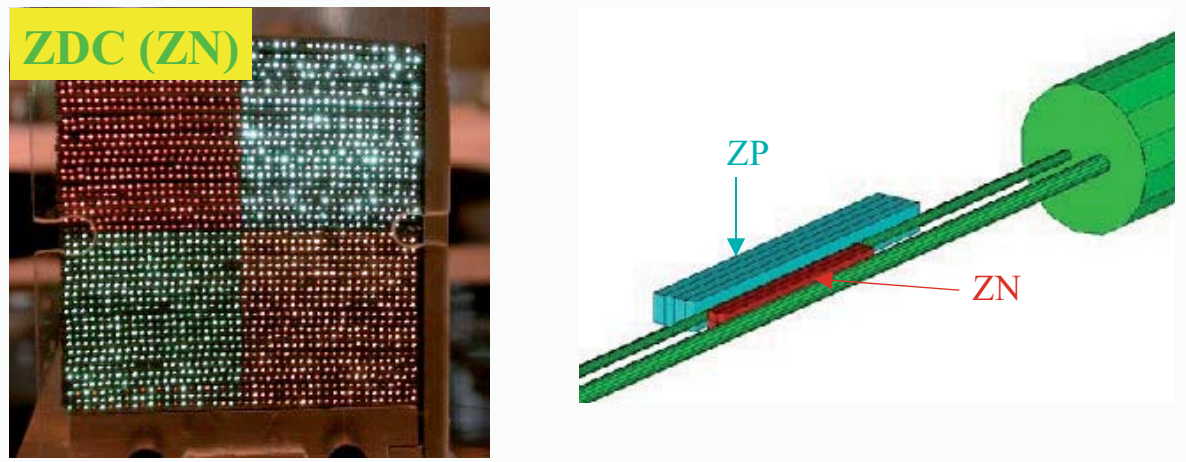

ense Matter in Heavy Ion Collisions and Astrophysics, A. Marin (Dubna July 2008)

20.09 .09

41

\section{Centrality determination in ALICE}

Event by event determination of the centrality:

Zero degree hadronic calorimeters (ZDC) +

electromagnetic calorimeters (ZEM)

EZDC , EZEM $\longrightarrow$ Nspec $\longrightarrow$ Npart $\longrightarrow$ Impact parameter (b)
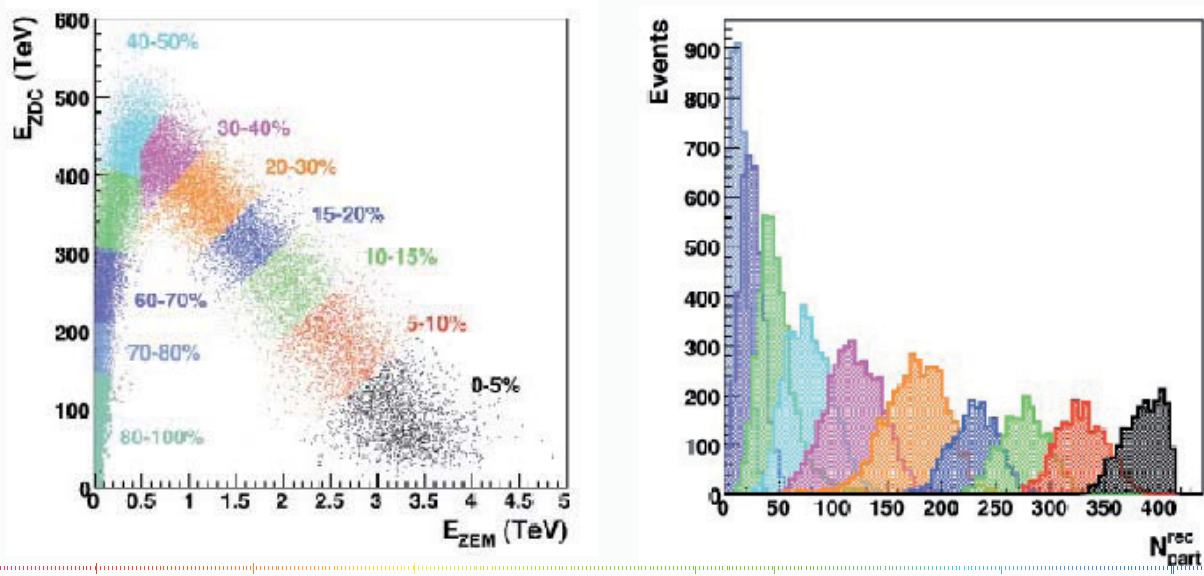

ense Matter in Heavy Ion Collisions and Astrophysics, A. Marin (Dubna July 2008)

20.09 .09

42 


\section{ALICE charged particle acceptance}

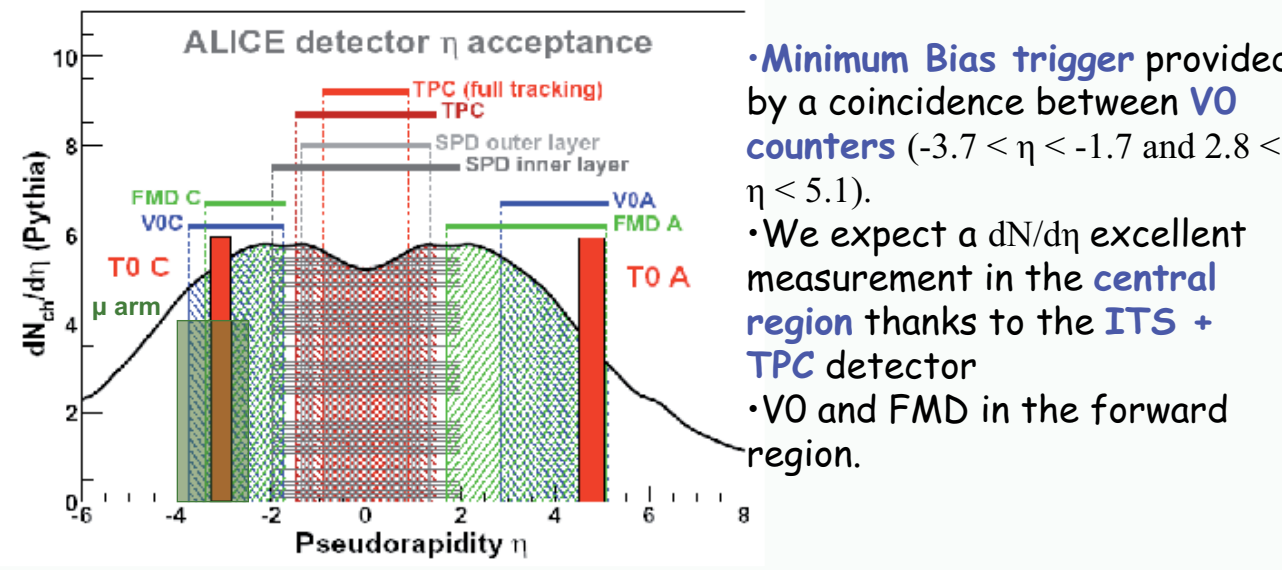

\section{The ALICE EXPERIMENT}

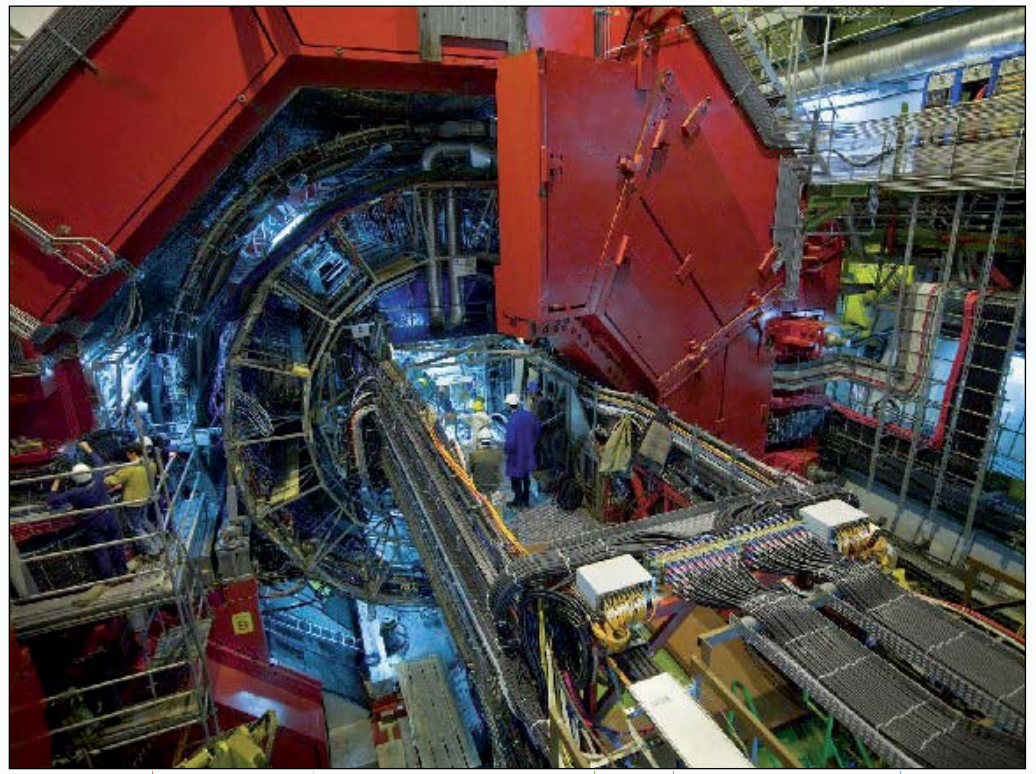

ense Matter in Heavy Ion Collisions and Astrophysics, A. Marin (Dubna July 2008) 


\section{ALICE - Global Tracking}

Parallel Kalman Filtering

After cluster finding, start iterative process through all central tracking detectors, ITS+TPC+TRD:

-Primary Vertex Finding in ITS

- Track seeding in outerTPC

-Propagation to the vertex, tracking in ITS

- Back-propagation in TPC and in the TRD

- Extrapolation and connection with outer PID detectors

- Final refit inwards

(for VO, 1-prong decays)

$d N / d y=8000$ (slice: $2^{\circ}$ in $\theta$ )

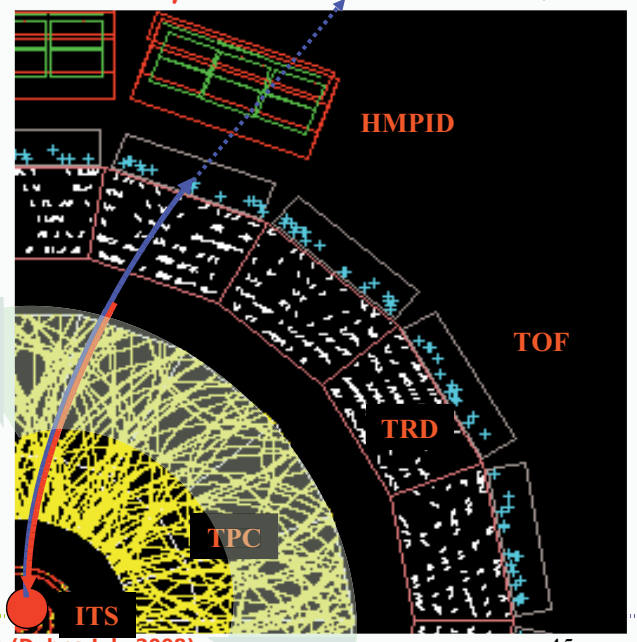

\section{Tracking/Physical efficiency}

Challenge in high-particle density environment

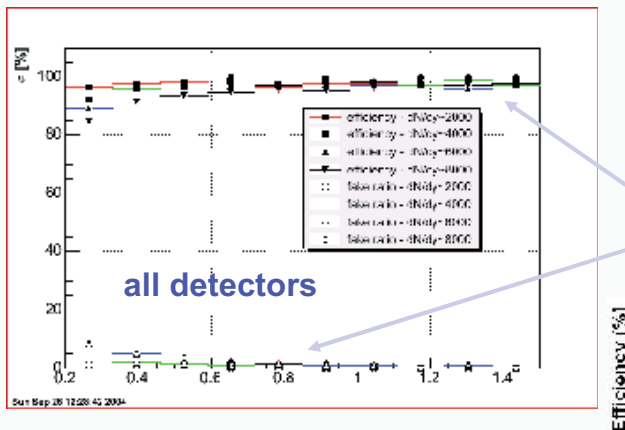

For realistic particle densities $\mathrm{dN} / \mathrm{dy}=2000-4000$ combined efficiency well above $90 \%$ and fake track probability below $5 \%$

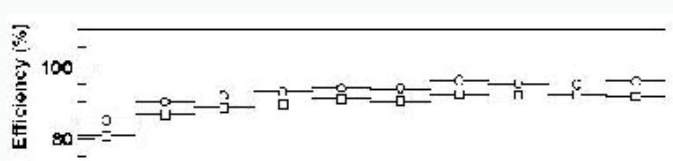

Efficiency normalized to number of generated particles at primary vertex within the central acceptance $|n|<0.9$ protons - large absorption kaons - decays on flight

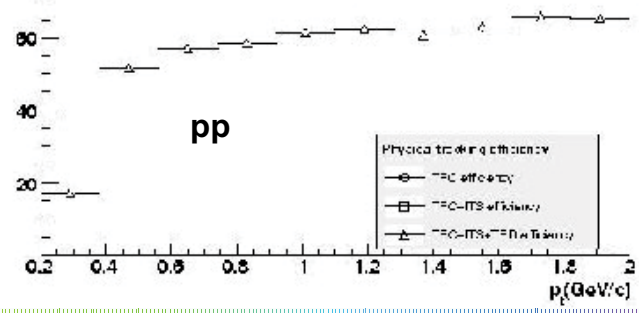




\section{Momentum resolution}

Robust, redundant tracking from $<100 \mathrm{MeV} / \mathrm{c}$ to $>100 \mathrm{GeV} / \mathrm{c}$ Very little dependence on $\mathrm{dN} / \mathrm{d} y$ up to $\mathrm{dN} / \mathrm{dy} \approx 8000$

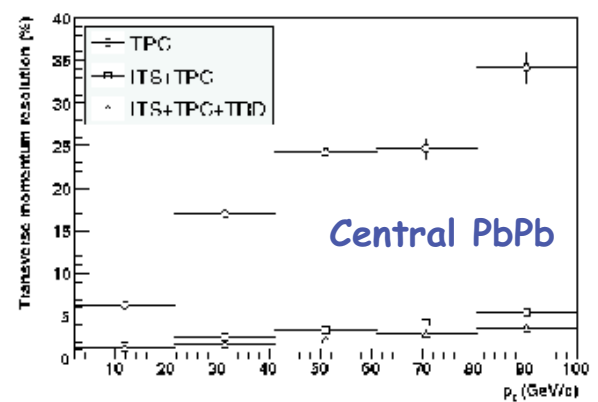

at low momentum dominated by

- ionization-loss fluctuations

- multiple scattering

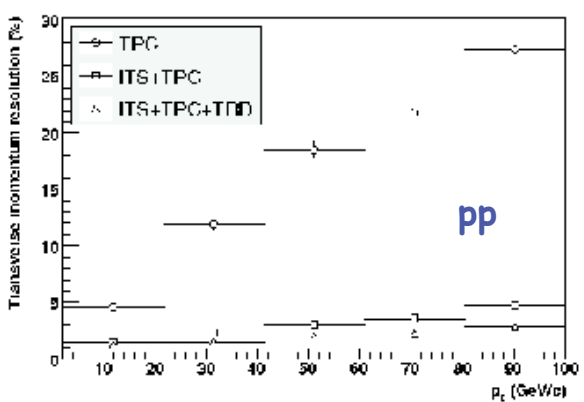

at high momentum determined by

- point measurement precision

- and the alignment \& calibration

\section{PID in ALICE}

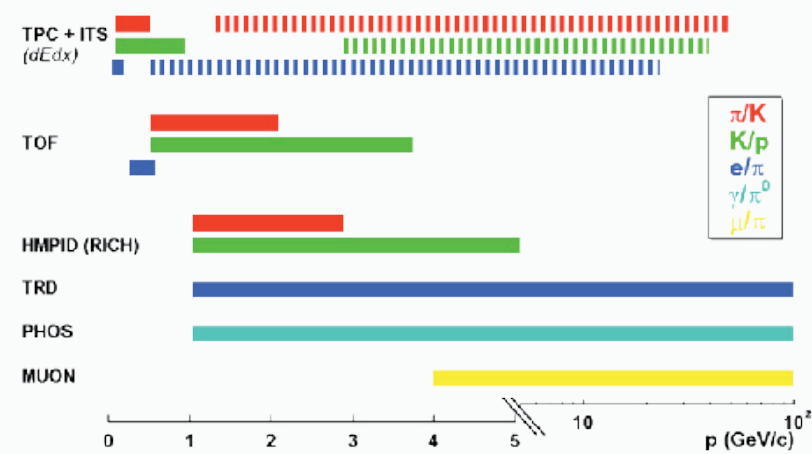

-excellent particle ID up to $~ 50$ to $60 \mathrm{GeV} / \mathrm{c}$

- Most $(2 \pi * 1.8$ units $\eta)$ of the hadrons ( $d E / d x+$ ToF), leptons ( $d E / d x$, TOF, transition radiation) and photons (high resolution EM calorimetry, conversions);

- Track and identify from very low (<100 MeV/c) up to very high pt (>100 GeV/c);

-Identify short lived particles (hyperons, D/B meson) through secondary vertex detection; 


\section{PID: Energy loss (dE/dx) in the TPC}

\section{Bethe-Bloch formula}

$$
\frac{\overline{\Delta E}}{\Delta x}=2 C \frac{m_{e} c^{2}}{\beta^{2}} \frac{Z z^{2}}{A} \rho \frac{\prod_{1}}{\mathrm{~K}^{2}} \ln \underset{\mathrm{X}}{\stackrel{\Phi 2 \gamma^{2} \beta^{2} m_{e} c^{2} E_{\max }}{I_{0}^{2}}} \stackrel{\mathrm{K}}{\mathrm{B}} \beta^{2}-\frac{\varepsilon}{2}-\frac{\delta(\beta)}{2} \stackrel{\mathrm{b}}{\mathrm{U}}
$$
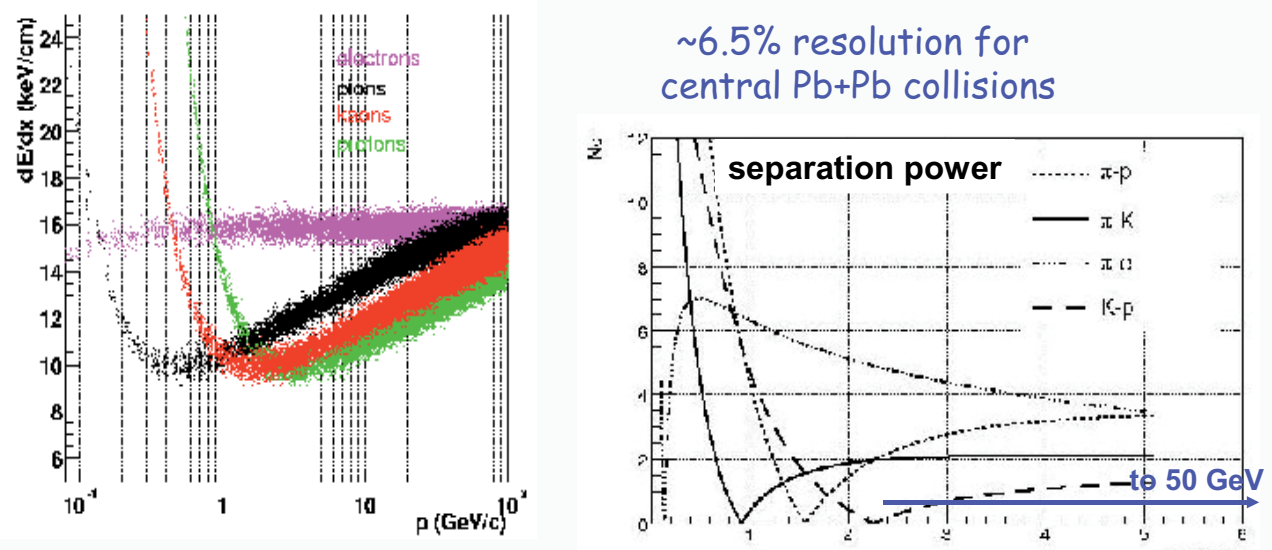

ense Matter in Heavy Ion Collisions and Astrophysics, A. Marin (Dubna July 2008)

20.09.09

49

\section{PID: dE/dx in the ITS}

- Based on specific ionization ( $d E / d x$ ) in the SDD and SSD (4 layers)

- Add information to the PID given by the TPC (combined-Bayesian PID)

- Identify tracks not reconstructed by the TPC:

- Low momentum

- Out of TPC acceptance

- Dead zones of TPC (between sectors)
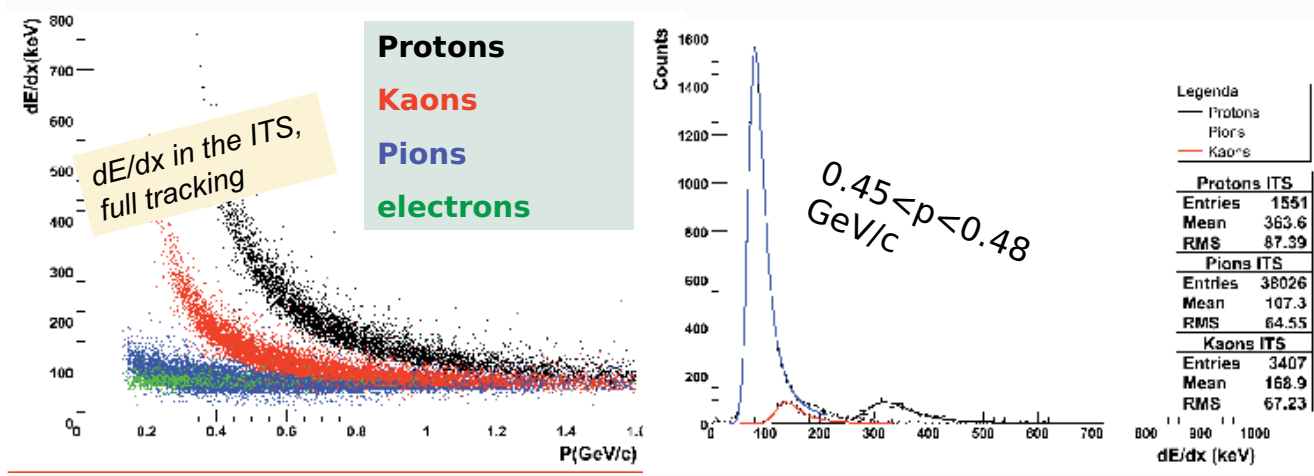

ense Matter in Heavy Ion Collisions and Astrophysics, A. Marin (Dubna July 2008)

20.09.09

$50 \quad 50$ 


\section{Mass separation with TOF}
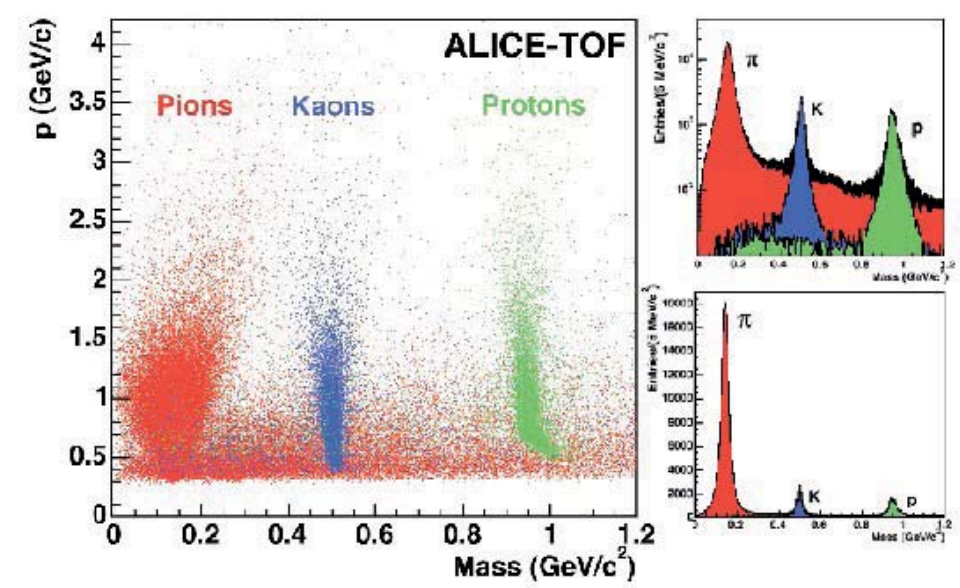

\section{PID: Pion efficiency with TRD}
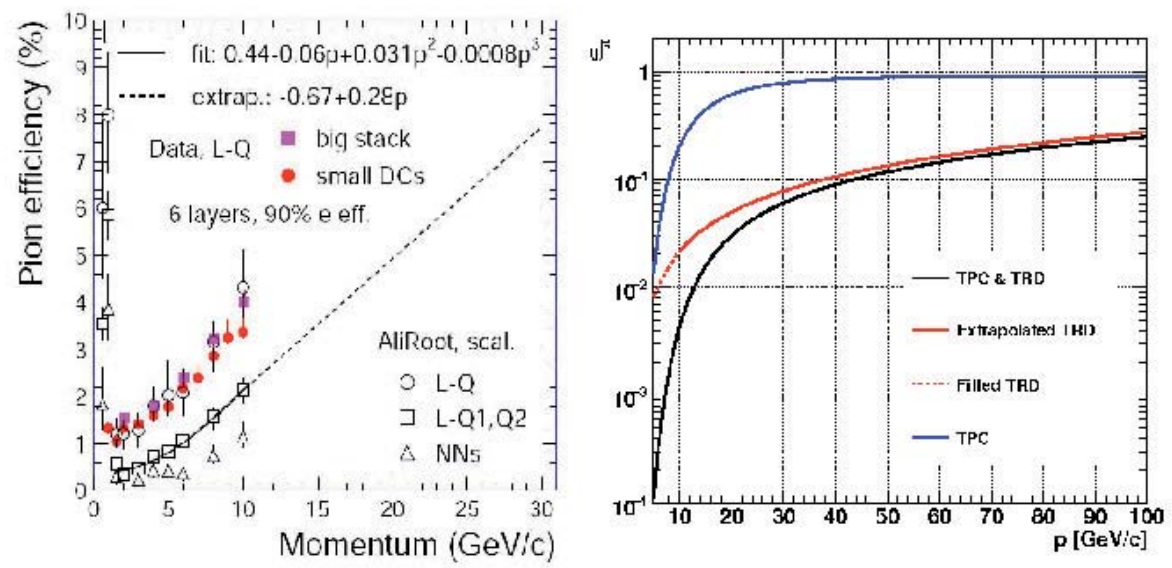


\section{Identified particle spectra}

Estimated $p_{+}$range for particle identification for $10^{7}$ central $\mathrm{Pb}$ $\mathrm{Pb}$ events (1-year data taking) $\pi, K, p: 0.1-0.15<p_{+}<50 \mathrm{GeV} / \mathrm{c}$

Weak or strong decaying particles: up to $10-15 \mathrm{GeV} / \mathrm{c}$

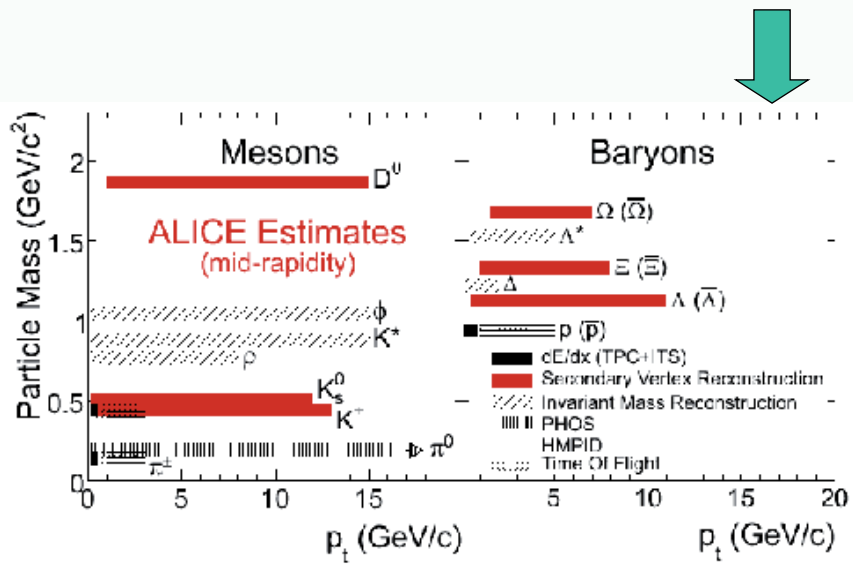

ense Matter in Heavy Ion Collisions and Astrophysics, A. Marin (Dubna July 2008)

20.09 .09

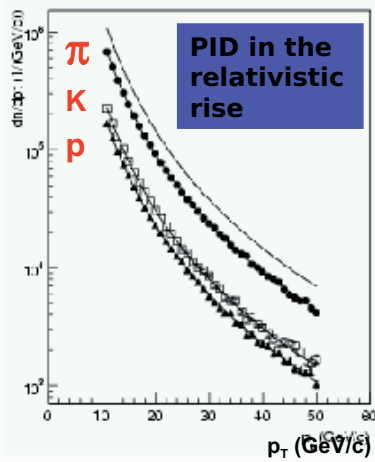

53

\section{ALICE running strategy}

- Regular pp runs at $14 \mathrm{TeV}$. First run at $10 \mathrm{TeV}$

- Initial heavy-ion programme:

- $\mathrm{Pb}-\mathrm{Pb}$ pilot run

- 1-2 years $\mathrm{Pb}-\mathrm{Pb}$

- 1 year PPb like collisions

- 1-2 years Ar-Ar

- Later options:

- dedicated pp at 5.5 TeV

- another intermediate mass A-A system

- possibly another pA system 


\section{ALICE running condlitions}

\begin{tabular}{|c|c|c|c|c|c|}
\hline Systim & 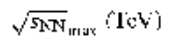 & $\Delta y$ & $\sigma_{\text {geom }}(b)$ & $f_{\text {low }}\left(\mathrm{CII}^{-2} \mathrm{~s}^{-1} \mathrm{j}\right.$ & $C_{\text {lugho }}\left(\mathrm{cmon}^{-2} \mathrm{~s}^{-1}\right)$ \\
\hline $\mathrm{Pb}-\mathrm{Th}$ & 5.5 & 0 & 7.7 & $1.0 \times 10^{2}$ & \\
\hline$A-A$ & 6.3 & 0 & 2.7 & $2.8 \times 10^{2 i}$ & $1.0 \times 10^{20}$ \\
\hline 00 & 7.0 & $\ddot{v}$ & 1.4 & $5.5,10^{2 i}$ & $2.0: 10^{29}$ \\
\hline$N-. N$ & 7.0 & 0 & 1.3 & $5.9 \times 10^{27}$ & $2.2 \times 10^{30}$ \\
\hline$\alpha \alpha$ & 7.0 & $\hat{a}$ & 0.34 & $6.2 \therefore 10^{20}$ & \\
\hline dત & 7.0 & 0 & 0.19 & $1.1 \times 10^{60}$ & \\
\hline $\mathrm{PP}$ & 14.0 & 0 & 0.07 & $1.0 \cdot 10^{29}$ & $5.0 ; 10^{\%}$ \\
\hline p'b & 8.8 & 0.47 & 1.9 & $1.1 \times 10^{20}$ & \\
\hline pAr & 9.4 & i.4i] & 门. 72 & $3.0 \times 10^{29}$ & \\
\hline $\left.\mathrm{l}^{6}\right)$ & 9.9 & 0.35 & 0.34 & $5.4 \times 10^{20}$ & \\
\hline $\mathrm{iFh}$ & 6.2 & 0.12 & 2.6 & $8.1 \therefore 10^{2 \mathrm{~B}}$ & \\
\hline dAr & 6.61 & 0.05 & 1.1 & $1.9 \times 10^{25}$ & \\
\hline 10 & 7.0 & 0.00 & 0.66 & $3.2=10^{20}$ & \\
\hline al'b & 6.2 & 0.12 & 2.75 & $7.7 \times 10^{28}$ & \\
\hline$\alpha A 1$ & 6.6 & 0.05 & 1.23 & $1.7 \cdot 10^{29}$ & \\
\hline$\alpha \mathrm{O}$ & 7.0 & 0.00 & 0.76 & $2.8 \times 10^{26}$ & \\
\hline
\end{tabular}

\section{LHC machine parameters}

Table 2.3. LHC machine parameress for $p p$ and $\mathrm{Fb}-\mathrm{Fb}$ runs for ALICE.

\begin{tabular}{|c|c|c|}
\hline & $\mathrm{pp}$ & $\mathrm{Fb}-\mathrm{Pb}$ \\
\hline Eucrgy per meleon (TcV) & 7 & 2.76 \\
\hline$\beta$ at the $\mathbb{F}: \beta^{-1}(\mathrm{~m})$ & 10 & 0.5 \\
\hline R.m.s. bean radius at IF: $\sigma_{1}(\mu \mathrm{m})$ & $71^{2}$ & 15.9 \\
\hline R.m.s. bunch lchgth: of (cm) & 7.7 & 7.7 \\
\hline \multicolumn{3}{|l|}{ Yertical cossing halt-angle ifaradj tor } \\
\hline pos. (nco.) $\mu$-spect. dipole polatization & 150150 & $150(100 ;$ \\
\hline Yo. of bunches & 2808 & 592 \\
\hline Builch spacing (us) & 24.95 & 99.8 \\
\hline Inicial number of particles per bunch & $1.1>10^{11}$ & $7.0 \because 10^{\circ}$ \\
\hline Inicial luminosity $\left(\mathrm{cm}^{-2} \mathrm{~s}^{-1}\right)$ & $\because 5 \times 10^{30}$ & $10^{27 \mathrm{~b}}$ \\
\hline
\end{tabular}

"For low-intensity runs $\beta^{\prime}$ could be $0.5 \mathrm{~m}$ and $\sigma_{\mathrm{c}}-1.59 \mathrm{~g} \mathrm{~m}$ as in l'b l'b.

" Larly operation will be with 62 bunches and $\sigma^{\prime}=1 \mathrm{~m}$, which yiclds an inicial lumimosity of $5.4 \times 10^{25} \mathrm{~cm}^{2} \leqslant 1$. 


\section{Soft Physics}

\section{Charged-particle Multiplicity Density}

Integrated multiplicity distributions from $\mathrm{Au}-\mathrm{Au} / \mathrm{Pb}-\mathrm{Pb}$ collisions and scaled pp collisions

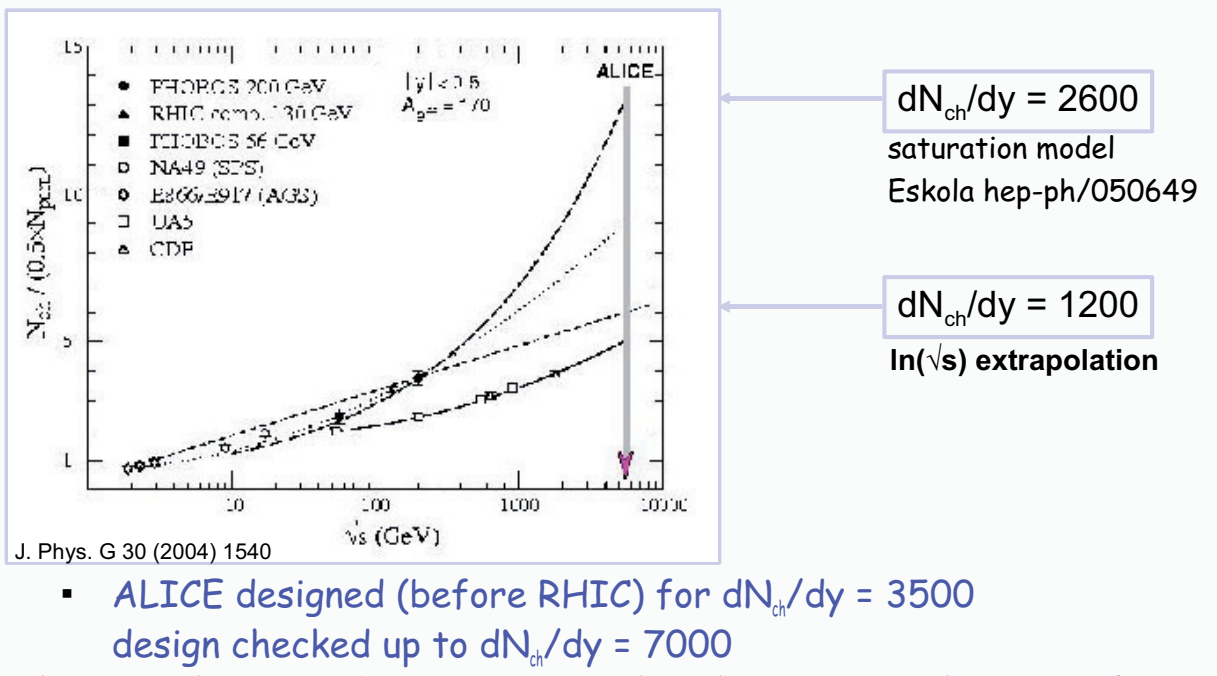




\section{Charged-particle Multiplicity Density}

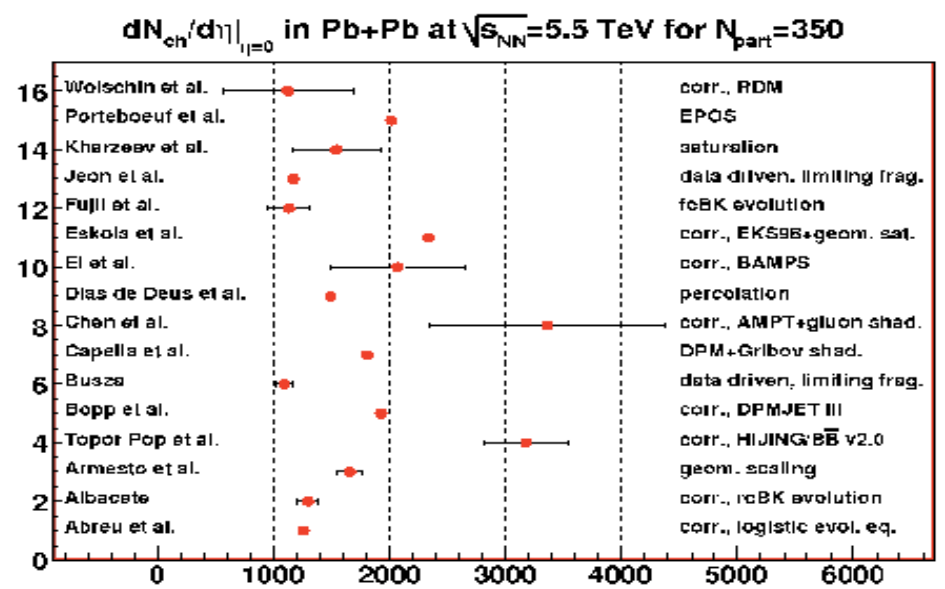

N. Armesto, arXiv:0804.4158.

LHC predictions: An overview

\section{Thermal Model and particle albundancies}

- Assume chemically equilibrated system at freeze-out (constant $T_{c h}$ and $\mu$ )

- Composed of non-interacting hadrons and resonances

- Given $T_{c h}$ and $\mu$ 's, particle abundances ( $\left.n_{i}^{\prime} s\right)$ can be calculated in a grand canonical ensemble

$$
n_{i}=\frac{g}{2 \pi^{2}} \int_{0}^{\infty} \frac{p^{2} d p}{e^{\left(E_{i}(p)-\mu_{i}\right) / T} \pm 1}, E_{i}=\sqrt{p^{2}+m_{i}^{2}}
$$

- Obey conservation laws: Baryon Number, Strangeness, Isospin

- Short-lived particles and resonances need to be taken into account 


\section{Equilibrium versus non equilibrium at LHC}

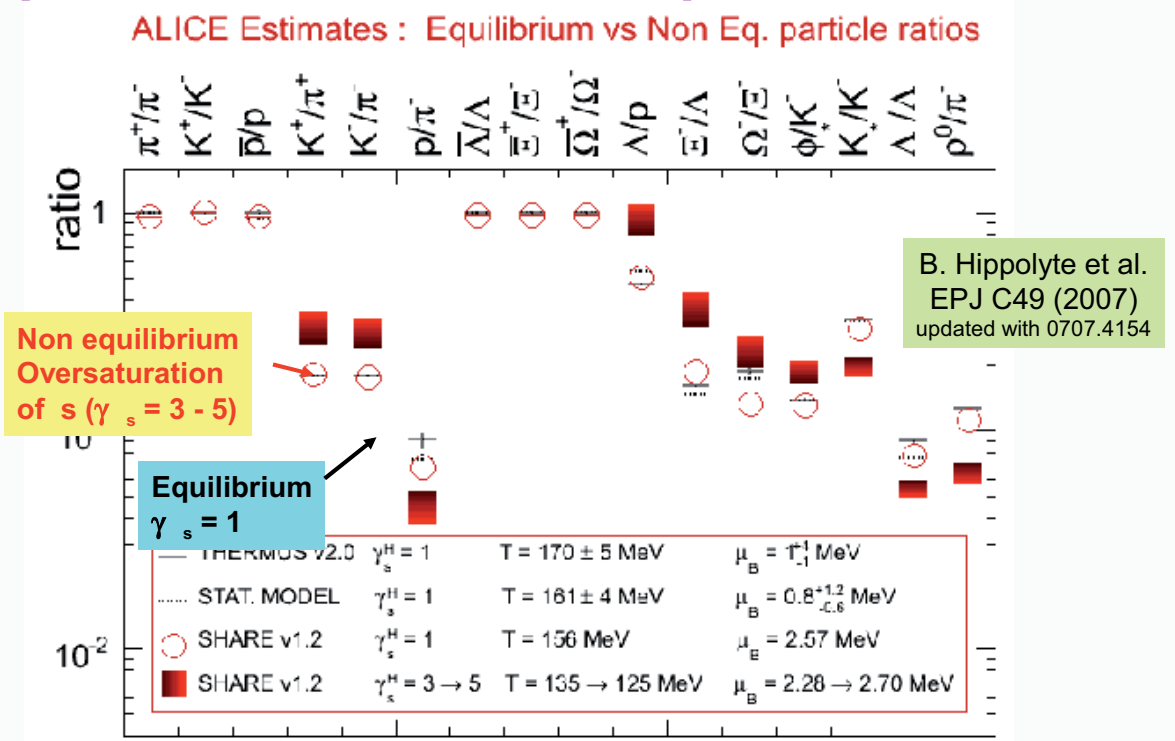

And many other questions: strangeness enhancement \% energy

Correlation volume ( $\mathrm{N}_{\text {part }} \rightarrow \mathrm{GC}, \mathrm{N}_{\text {bin }} \rightarrow$ hard processes) ? Evolution in pp ?

ense Matter in Heavy Ion Collisions and Astrophysics, A. Marin (Dubna July 2008) 20.09.09

61

\section{Resonances $\left(\rho, \varphi, K^{*}, \ldots\right)$}

Short-lived resonances:

- Time difference between chemical and kinetic freeze-out

- In medium modifications of mass, width, comparison between hadronic and leptonic channels $\rightarrow$ partial chiral symmetry restoration

- Reconstruction by invariant mass spectrum, background subtracted (like-sign method).

- Mass resolutions $\sim 1.5-3 \mathrm{MeV}$. $\mathrm{p}_{\mathrm{T}}$ stat. limits from $8(\rho)$ to $15 \mathrm{GeV} / \mathrm{c}\left(\varphi, \mathrm{K}^{*}\right)$
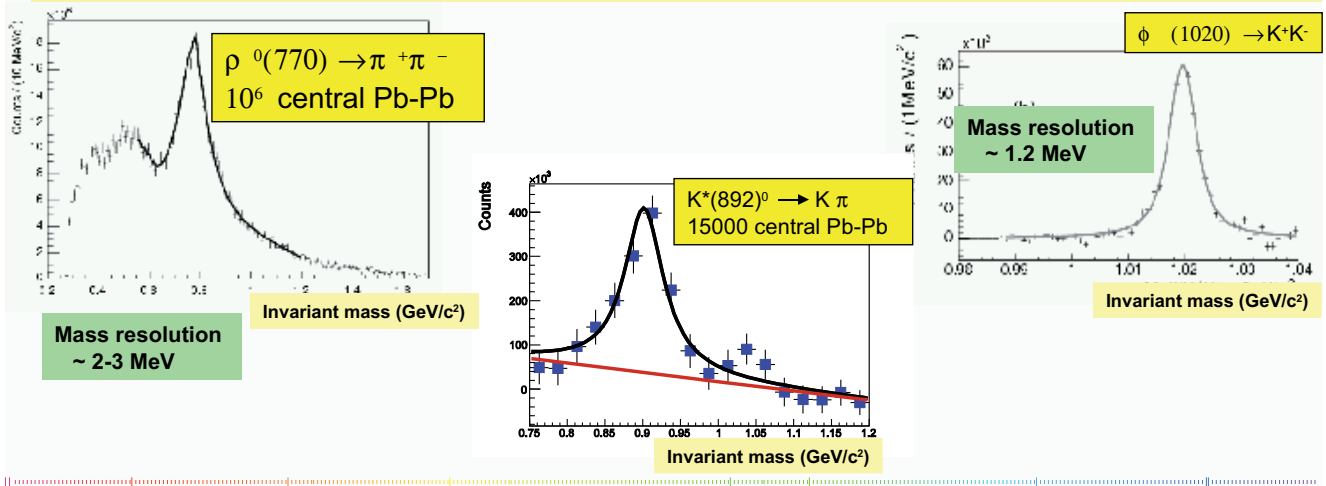


\section{Reaction plane}

- The initial spatial anisotropy is transformed into momentum anisotropy

-Analyze in terms of response of the system to initial pressure

- Deduce (model) thermalizing time, EOS

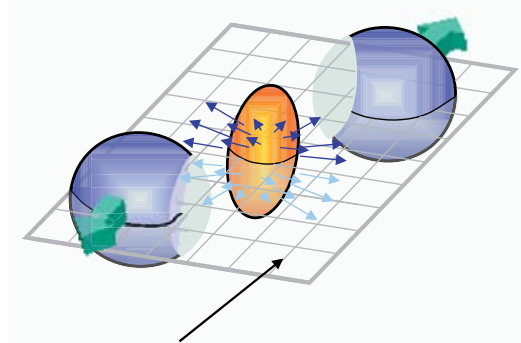

reaction plane

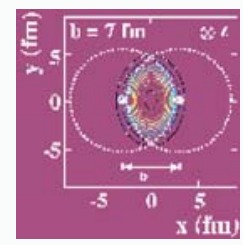

20.09.09

\section{Energy dependence of flow}

eccentricity vs. particle multiplicity in overlap region

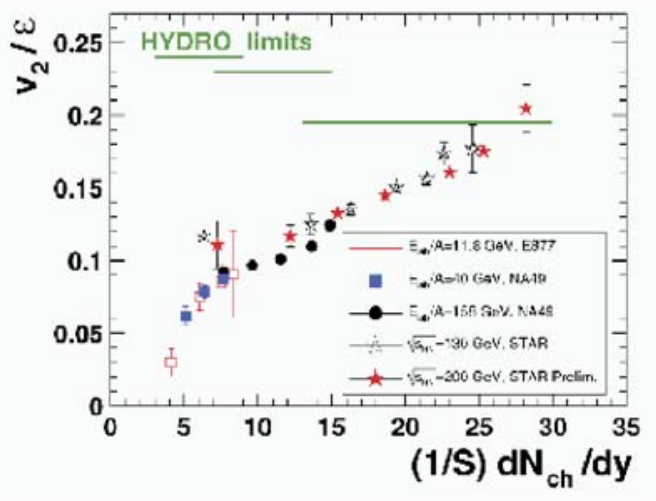

hydrodynamical limit reached at RHIC

$\rightarrow$ 'ideal fluid'

- clear predictions from hydrodynamics

-sensitive to equation-of-state 


\section{Predictions of $\mathrm{v}_{2}$ for LHC}
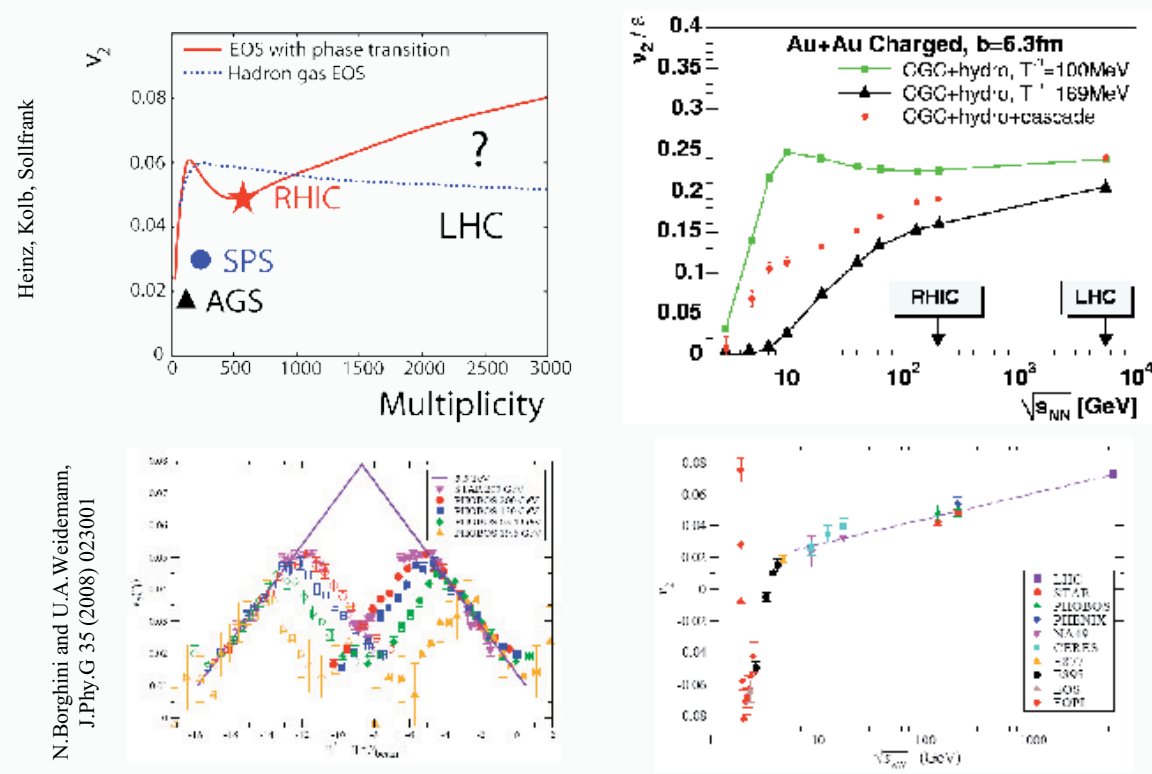

\section{Heavy quarks and Quarkonia}




\section{Charm and beauty at LHC}

- Measure parton energy loss in QGP

- Test of PQCD

- Cross sections needed to understand quarkonia production in HIC

MNR code (NLO): Mangano, Nason, Ridolfi, NPB373 (1992) 295.

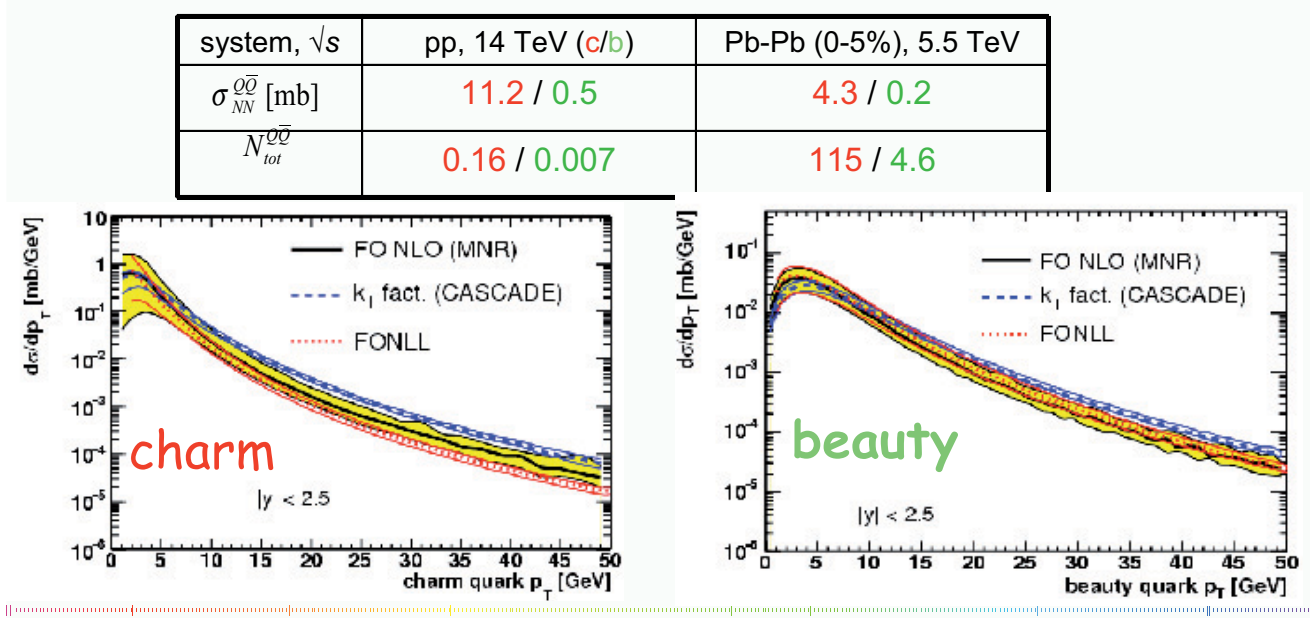

ense Matter in Heavy Ion Collisions and Astrophysics, A. Marin (Dubna July 2008) 20.09.09

67

\section{Heavy quarks as medium probes: Energy Loss}

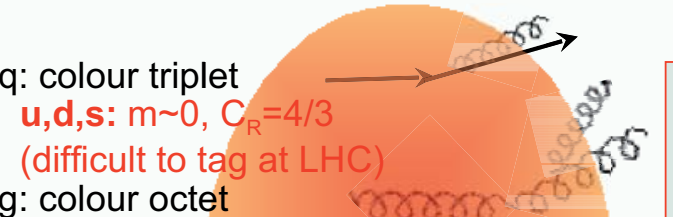

\section{Parton Energy Loss by}

- medium-induced gluon radiation - collisions with medium gluons

g: $\quad m=0, C_{R}=3$

$>$ E loss, dominant at $\mathrm{LHC}$

Q: colour triplet
c: $m \sim 1.5 \mathrm{GeV}, \mathrm{C}_{\mathrm{R}}=4 / 3$
small $m$, tagged by $D$ 's
b: $m \sim 5 \mathrm{GeV}, \quad \mathrm{C}_{\mathrm{R}}=4 / 3$
large mass, < Eloss

$$
\Delta E\left(\varepsilon_{Q G P} ; C_{R}, m, L\right)
$$
pred: $\Delta E_{g}>\Delta E_{c \approx q}>\Delta E_{b}$

$$
\rightarrow R_{A A}^{\pi}<R_{A A}^{D}<R_{A A}^{B}
$$

\section{QCD medium}

Dokshitzer and Kharzeev, PLB 519 (2001) 199. Armesto, Salgado, Wiedemann, PRD 69 (2004) 114003.

Djordjevic, Gyulassy, Wicks, nucl-th/0512076 


\section{Physics Analysis}

- Hadronic decays:

$-\mathbf{D}^{0} \rightarrow \mathbf{K}^{-} \pi+, \mathbf{D}^{+-} \rightarrow \mathbf{K}^{-+} \pi{ }^{+} \pi{ }^{+-}, \mathbf{D}_{\mathbf{s}} \rightarrow \mathbf{K} \mathbf{K}^{*}, \mathbf{D}_{\mathbf{s}} \rightarrow \phi$ $\pi, \ldots$

- Leptonic decays:

- Single lepton analysis:

- Open charm, open beauty, electro-weak bosons:

- Invariant mass analysis of lepton pairs:

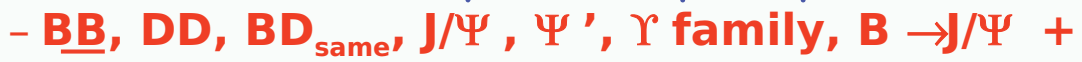
anything;

- Multi-lepton analysis :

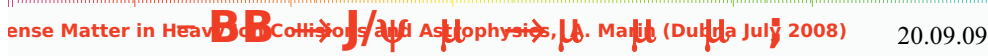

\section{Charm reconstruction: $\mathrm{D}^{0} \rightarrow \mathrm{K}^{-} \pi+$}

- Large combinatorial background ( $d N_{c h} / d y=6000$ in central Pb-Pb!)

- Main selection: displaced-vertex selection

- pair of opposite-charge tracks with large impact parameters

- good pointing of reconstructed Do momentum to the primary vertex

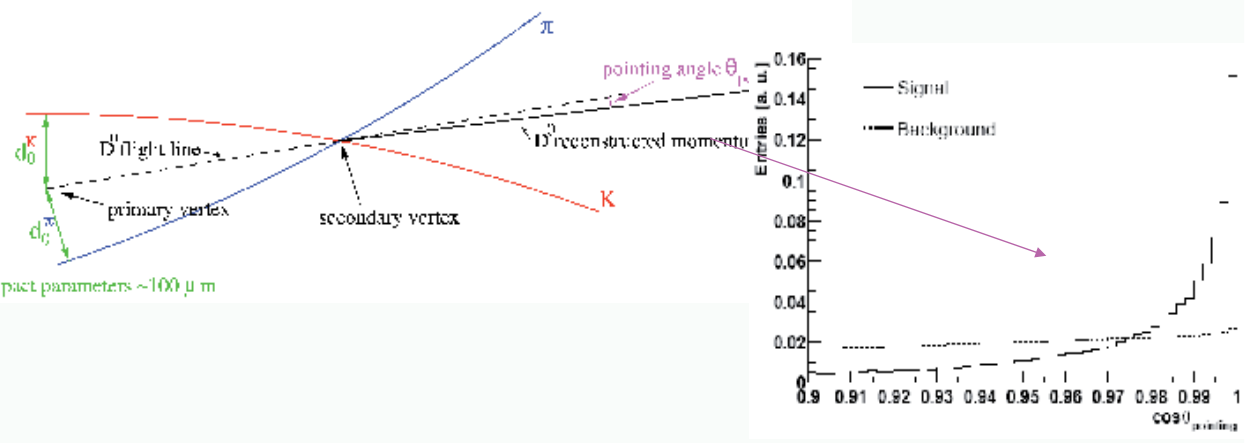




\section{$D^{0}$-meson Performance}
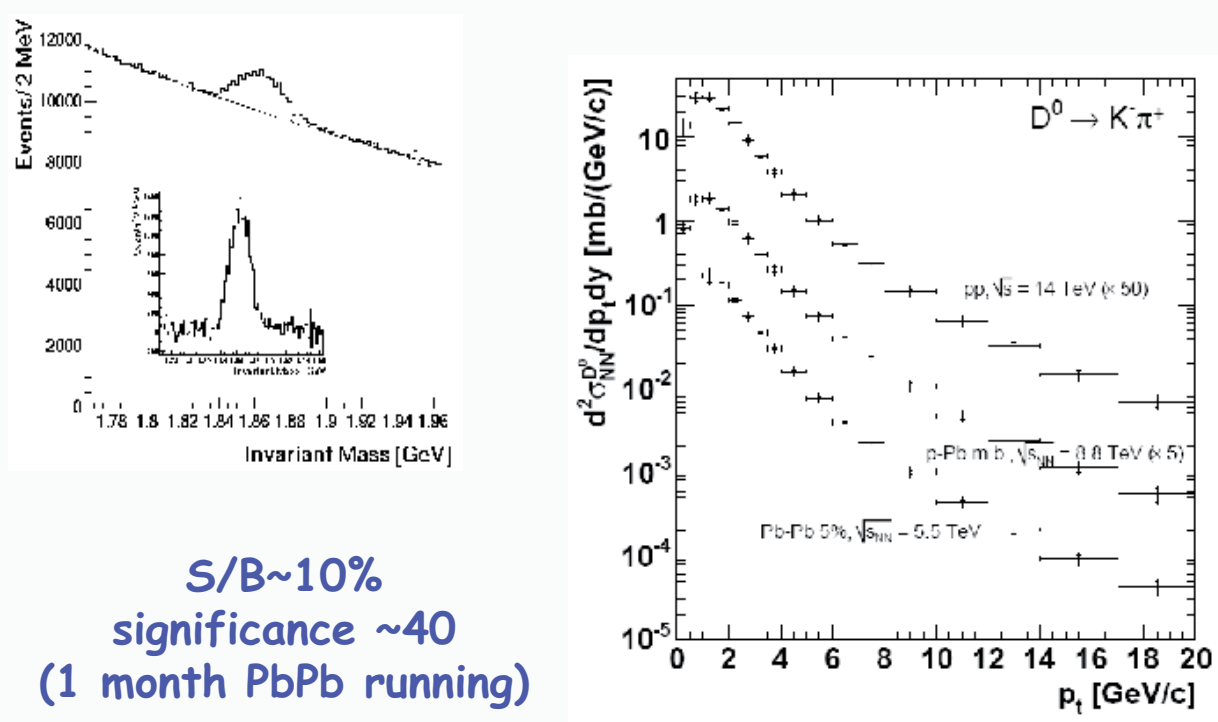

\section{Beauty via displaced electrons}

- ALICE has excellent electron identification capabilities

- Displaced electrons from B decays can be tagge Primary by an impact parameter cut

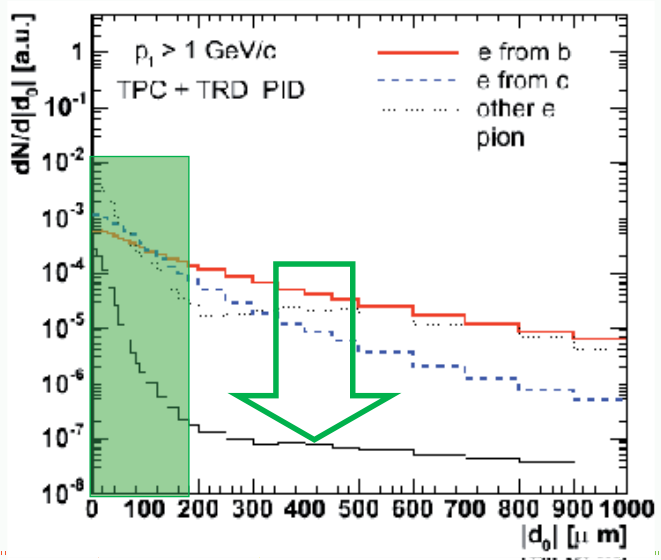




\section{Beauty via displaced $\mathrm{J} / \Psi$}

- ALICE has excellent electron identification capabilities

- Displaced di-electrons from B decays can be tagged by an impact parameter cut

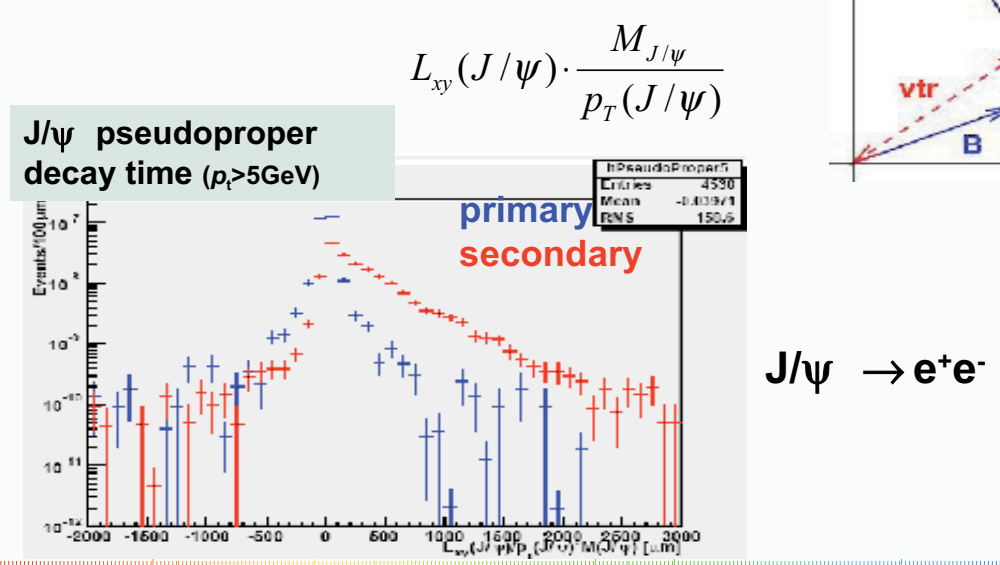

20.09 .09

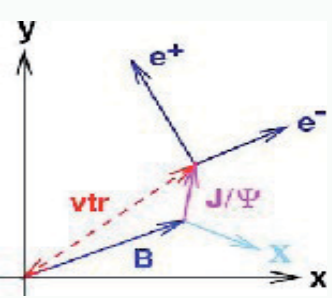

ense Matter in Heavy Ion Collisions and Astrophysics, A. Marin (Dubna July 2008)

\section{Expected $p_{t}$ coverage}
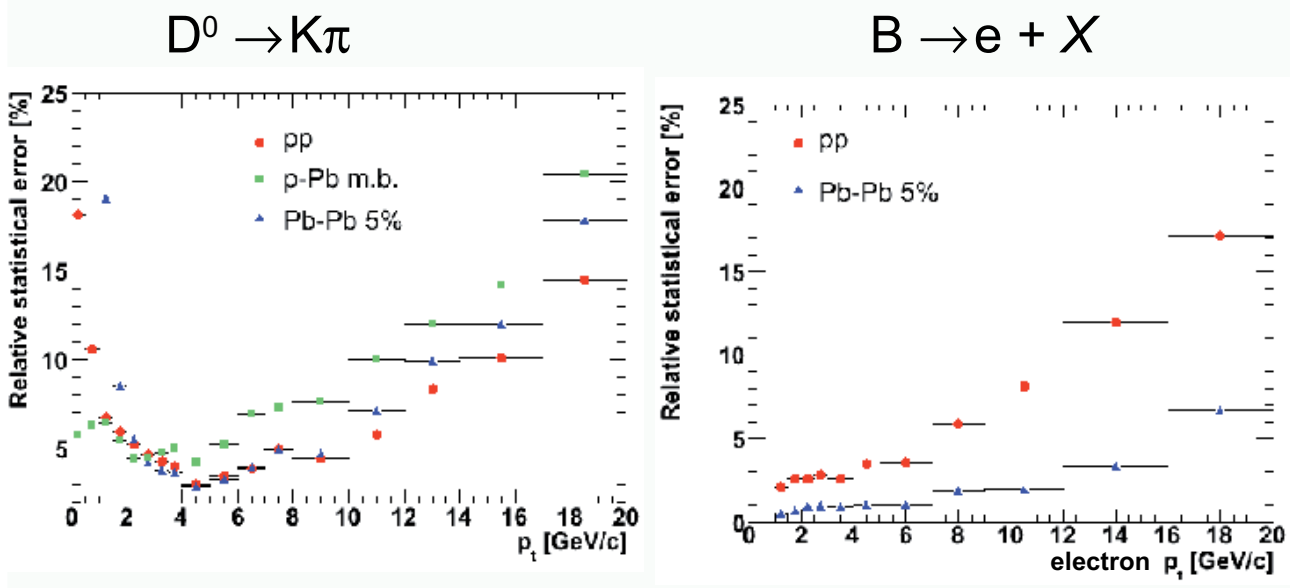

1 year at nominal luminosity

(107 central $\mathrm{Pb}-\mathrm{Pb}$ events, $10^{9} \mathrm{pp}$ events) 


\section{Calibrating the probe $(p p, \sqrt{s}=14 \mathrm{TeV})$

$$
R_{A A}\left(p_{t}\right)=\frac{1}{\left\langle N_{\text {coll }}\right\rangle} \times \frac{\mathrm{d} N_{A 4} / \mathrm{d} p_{t}}{\mathrm{~d} N_{p p} / \mathrm{d} p_{t}} \quad \begin{aligned}
& \text { measured at } 14 \mathrm{TeV} \text {, then } \\
& \text { scaled by pQCD to } 5.5 \mathrm{TeV}
\end{aligned}
$$

Expected sensitivity in comparison to PQCD:

$$
\mathrm{D}^{0} \rightarrow \mathrm{K} \pi
$$

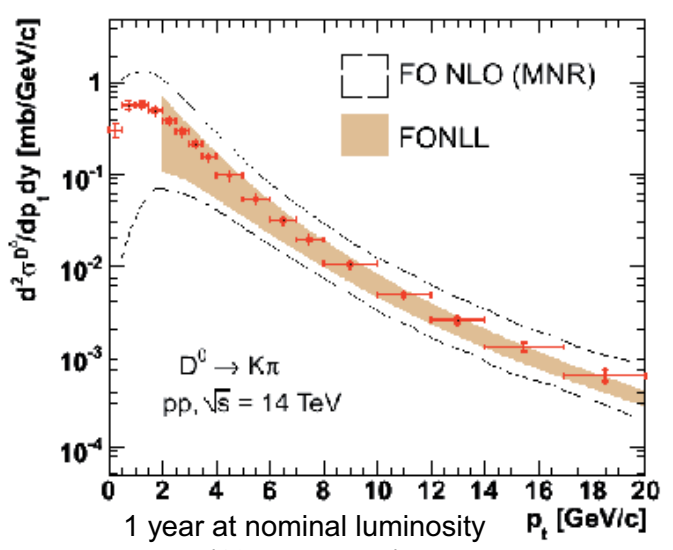

ense Matter in Heavy lon ed events) $\left(10^{9}\right.$ evions and Astrophysics, A. Marin (Dubna July 2008) 20.09 .09

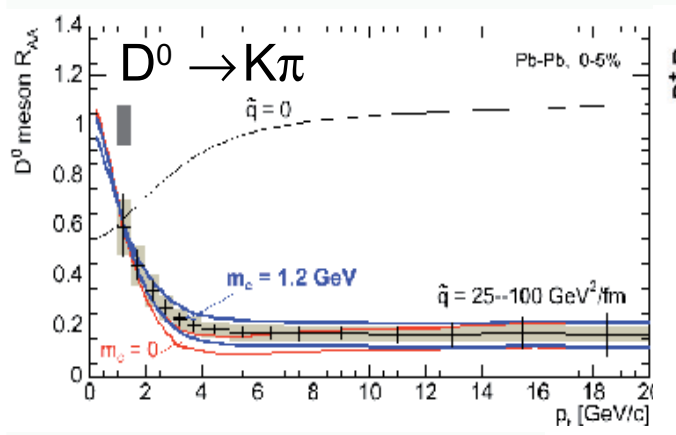

$\mathrm{B} \rightarrow \mathrm{e}+X$

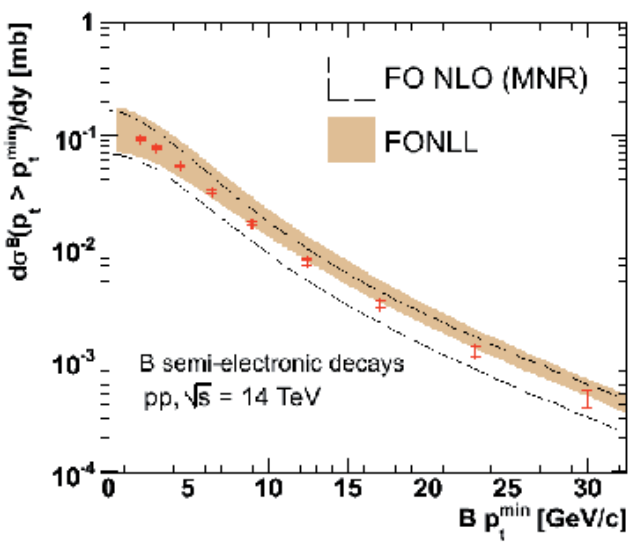

75

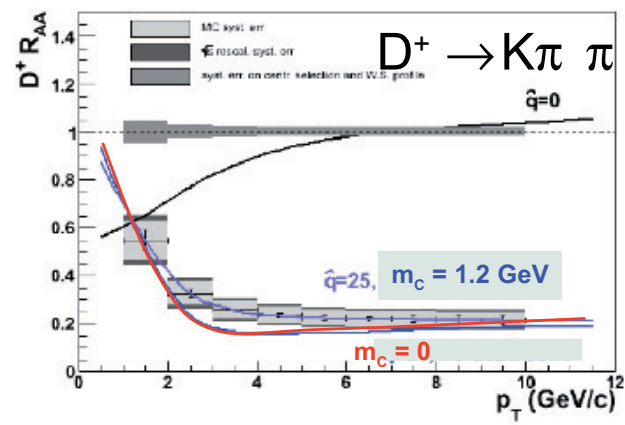

E loss calc.:

Armesto, Dainese, Salgado, Wiedemann

1 year at nominal luminosity (107 central $\mathrm{Pb}-\mathrm{Pb}$ events, $10^{9} \mathrm{pp}$ events)

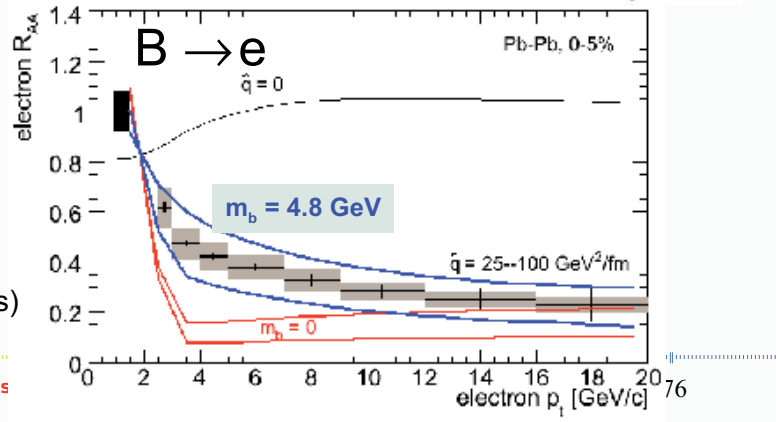

ense Matter in Heavy Ion Collisions and Astrophysics 


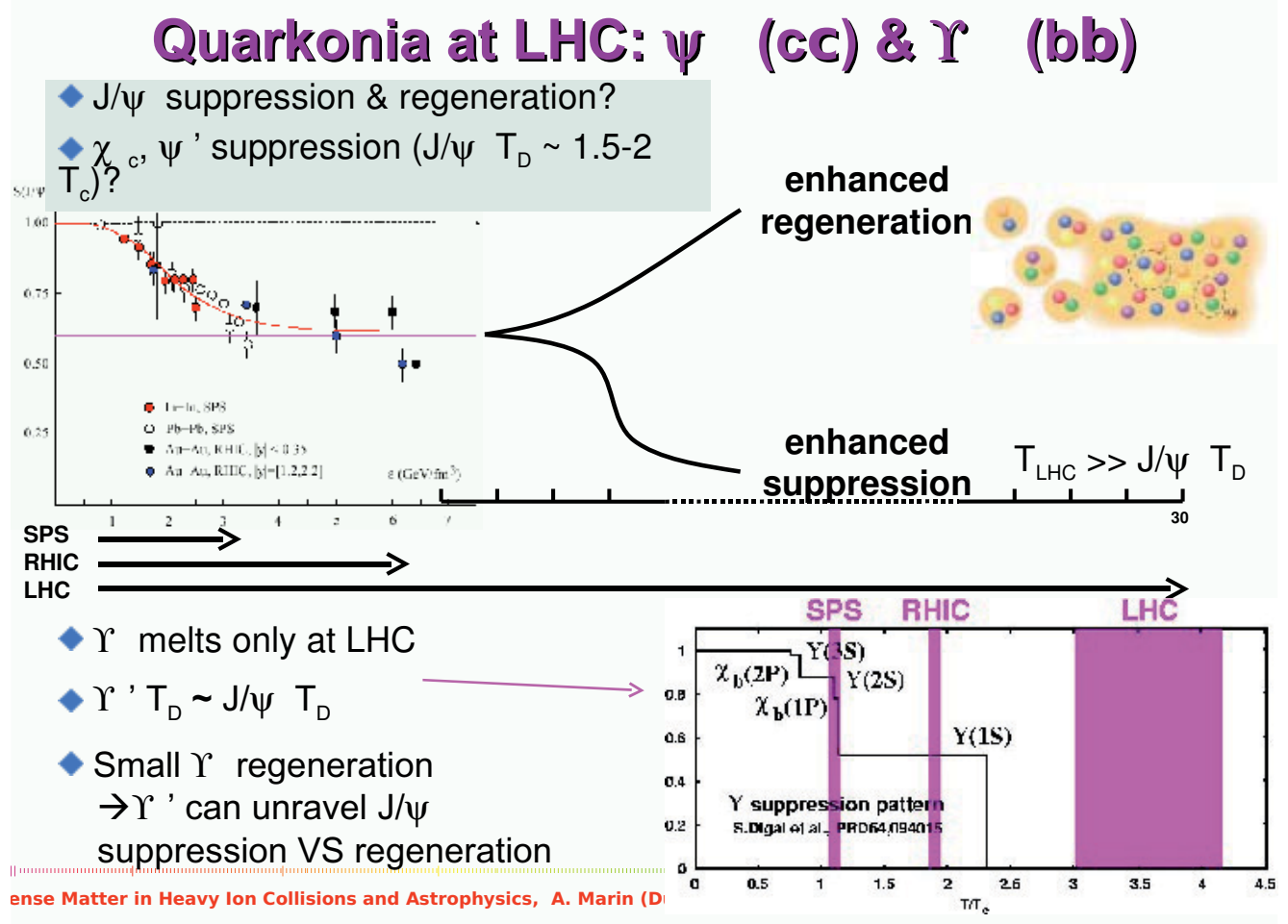

\section{Statistical Hadronization Modlel (PLB 652 (2007) 259)}
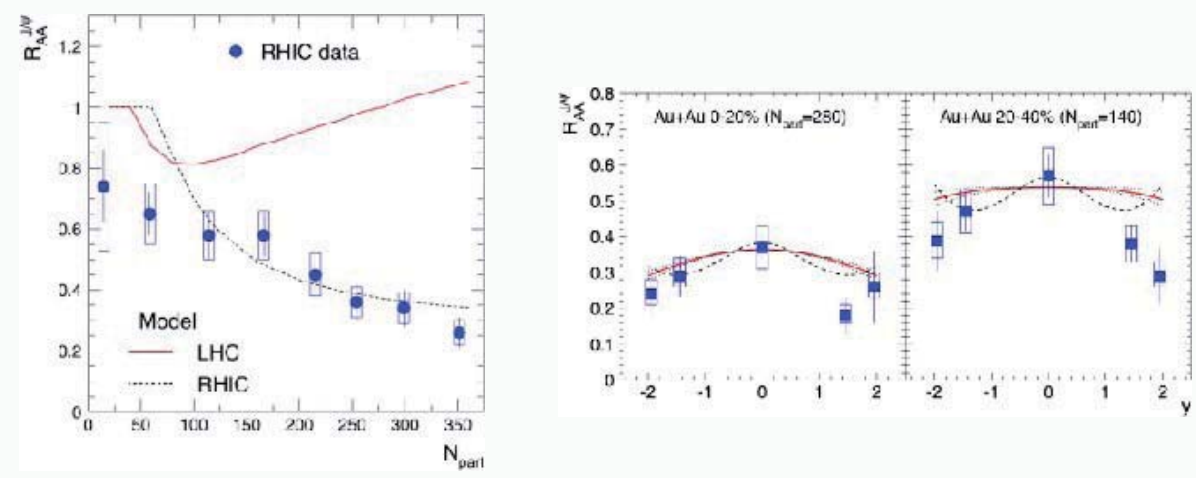

Clear signal for generation of charmonia due to statistical hadronization at the phase boundary 


\section{Quarkonia in the electron channel}

- Electron Identification (TRD+TPC);

- Tracking: ITS+TPC+TRD;

- $2 \times 10^{8}$ central PbPb events (no e-trigger).

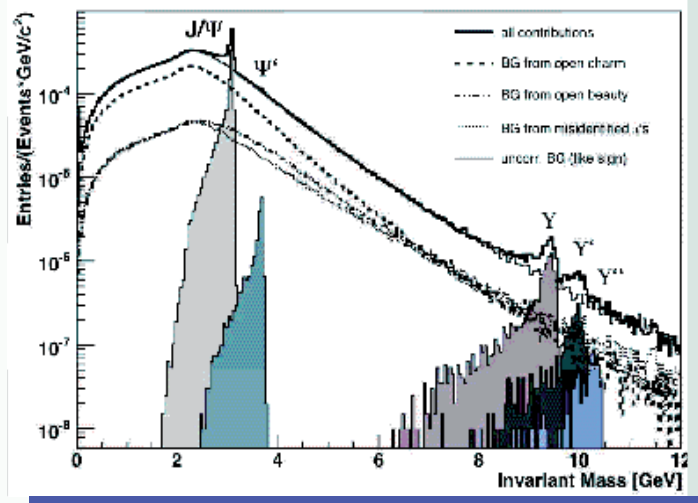

\section{$10 \%$ central $\mathrm{PbPb}$ dNch/dy $=3000$}
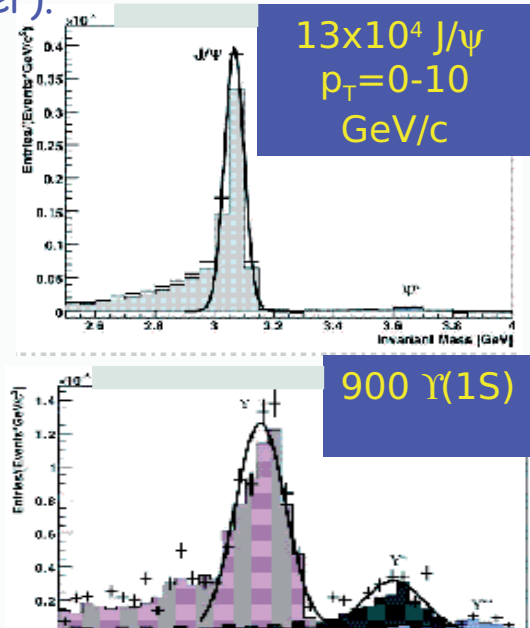

$900 \Upsilon(1 S)$

ense Matter in Heavy Ion Collisions and Astrophysics, A. Marin (Dubna July 2008)

20.09 .09

\section{Quarkonia in the muon channel}

\section{$\mathrm{PbPb}$ cent, $0 \mathrm{fm}<\mathrm{b}<3 \mathrm{fm}$}
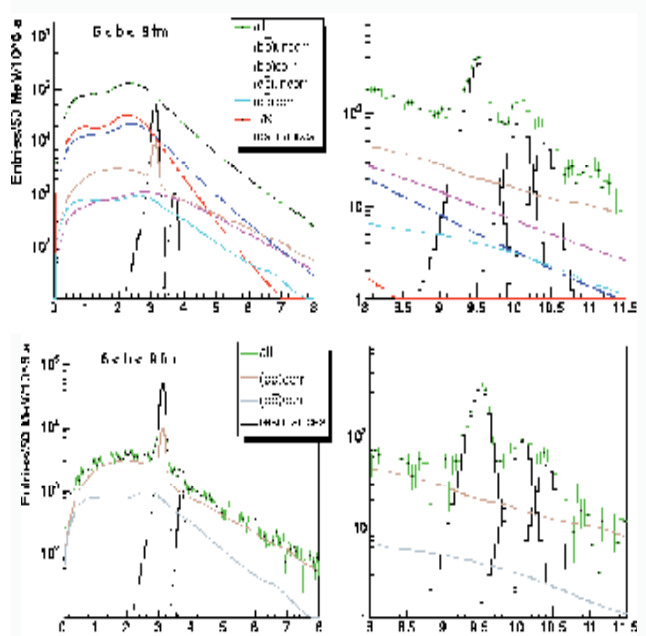

\begin{tabular}{|c|c|c|c|c|}
\hline State & $\mathrm{S}\left[10^{3}\right]$ & $\mathrm{B}\left[10^{3}\right]$ & $\mathrm{S} / \mathrm{B}$ & $\mathrm{S} /(\mathrm{S}+\mathrm{B})^{1 / 2}$ \\
\hline $\mathrm{J} / \Psi$ & 130 & 680 & 0.20 & 150 \\
\hline$\Psi^{\prime}$ & 3.7 & 300 & 0.01 & 6.7 \\
\hline$\Upsilon(1 \mathrm{~S})$ & 1.3 & 0.8 & 1.7 & 29 \\
\hline$\Upsilon(2 \mathrm{~S})$ & 0.35 & 0.54 & 0.65 & 12 \\
\hline$\Upsilon(3 \mathrm{~S})$ & 0.20 & 0.42 & 0.48 & 8.1 \\
\hline
\end{tabular}

Yields for baseline

- $\Upsilon(1 \mathrm{~S}) \& \Upsilon(2 \mathrm{~S}): 0-8 \mathrm{GeV} / \mathrm{c}$

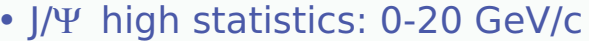

- $\Psi^{\prime}$ poor significance

- $\Upsilon$ (3S) ok, but 2-3 run needed. 


\section{Quarkonia Performance}

Bkg input: $\mathrm{dN}_{\mathrm{ch}} / \mathrm{dy}=4000$ in central Pb-Pb. $S / \sqrt{S+B}$ for 1 month

\begin{tabular}{|c|c|c|c|c|}
\hline Particle & $\begin{array}{c}\text { Charmonia } \\
u^{+} u^{-}\end{array}$ & Charmonia $e^{+} e^{-}$ & Bottonia $\mu{ }^{+} \mu$ & Bottonia $e^{+} e^{-}$ \\
\hline $\begin{array}{l}\text { Bkg- } \\
\text { subtracted } \\
\text { mass } \\
\text { plot }\end{array}$ & & + & & $\sqrt{15}$ \\
\hline $\begin{array}{c}\text { acc. } \\
\eta\end{array}$ & $-\int_{3}^{f} \underbrace{(5}_{3.5}$ & 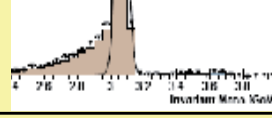 & $8.5 \quad 999.5 \quad 10 \quad 10.5$ & rsartiven \\
\hline M res. & $65 \mathrm{MeV}$ & $35 \mathrm{MeV}$ & $90 \mathrm{MeV}$ & $90 \mathrm{MeV}$ \\
\hline$S$ & $\mathrm{~J} / \psi 150, \psi^{\prime} 7$ & $\mathrm{~J} / \psi 245$ & 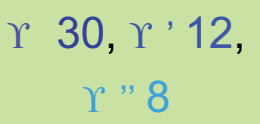 & $\Upsilon 21, \Upsilon 8$ \\
\hline$\overline{\sqrt{S+B^{-B}}}$ & $\psi, ? \psi^{\prime}$ & $\psi, ? ? \psi^{\prime}$ & $\Upsilon \mathfrak{x}, ? \Upsilon^{\prime \prime}$ & 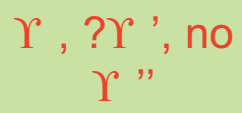 \\
\hline$p_{\mathrm{t}}$ & $\mathrm{J} / \psi \quad 0-20 \mathrm{GeV}$ & $\begin{array}{c}\mathrm{J} / \psi \quad 0-10 \\
\text { GeV }\end{array}$ & $\Upsilon 0-8 \mathrm{GeV}$ & -- \\
\hline
\end{tabular}

\section{Photons, Jets and high $p_{T}$}




\section{Direct photon}

Why direct photons?: Exit medium unscattered

Carry information on the entire history of the collision

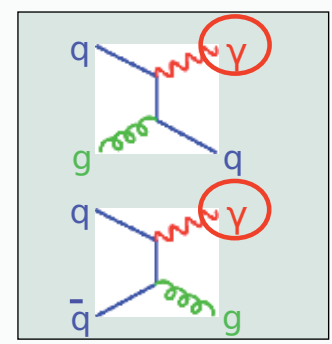

qg Compton Scattering

qq

Annihilation

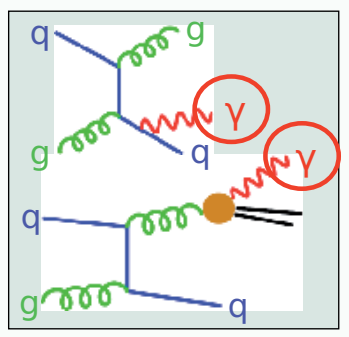

Bremsstrahlung

Thermal photons from QGP and hadron gas

Photons from Jet re-interaction in the medium $\ldots$ in a large background from $\pi^{\circ}$ and $\eta$ decays

\section{Photon sources in A-A collisions at LHC}

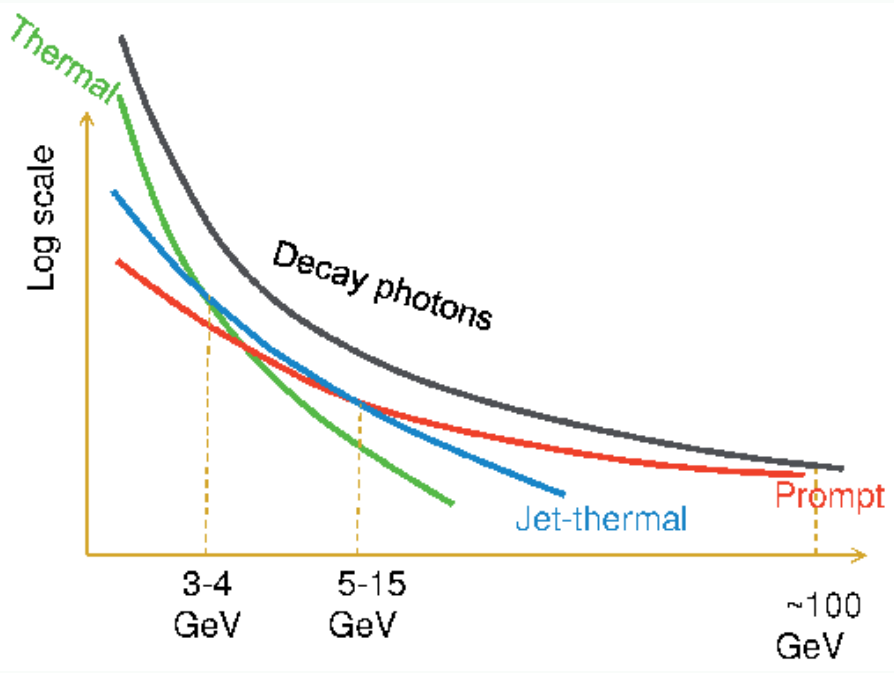




\section{How many direct photons,jets?}

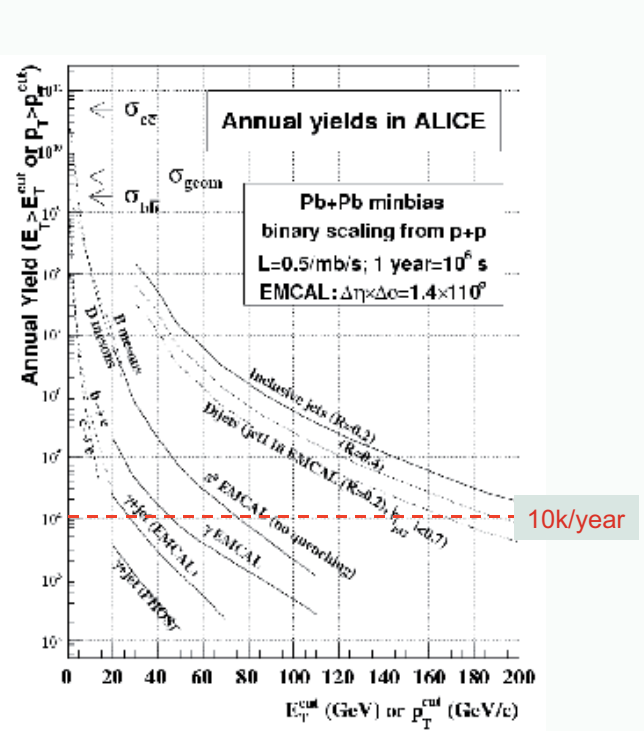

Large sample of direct LO $\gamma$-jet for $\mathrm{P}_{\mathrm{T}}<$ $30 \mathrm{GeV} / \mathrm{c}$ in PHOS and $p_{\mathrm{T}}<50 \mathrm{GeV} / \mathrm{c}$ in EMCal ...and in central Barrel

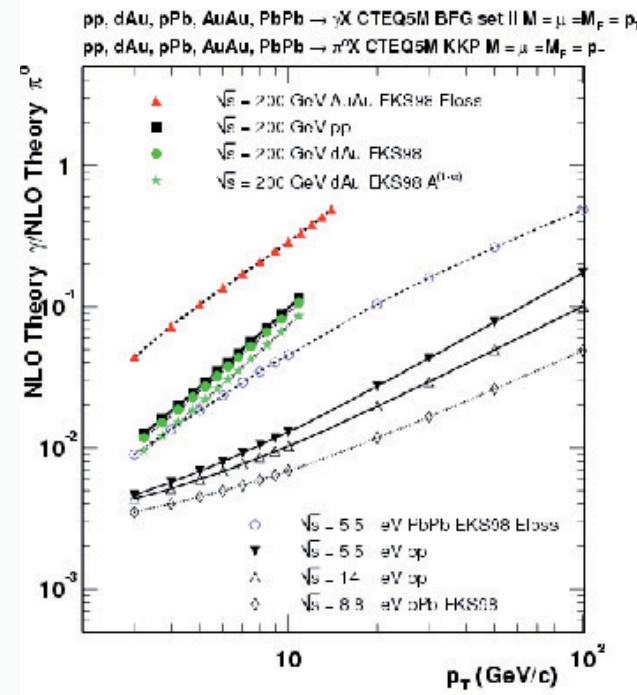

... but $\gamma / \pi^{0}=0,01-0,1$ for $\mathrm{p}_{\mathrm{T}}>20 \mathrm{GeV} / \mathrm{c}$ We need a good $\gamma / \pi$ OPID Marin (Dubna July 2008) $\quad 20.09 .09$

\section{Photon Production: different sources}

Turbide, Gale, Jeon, and

Moore
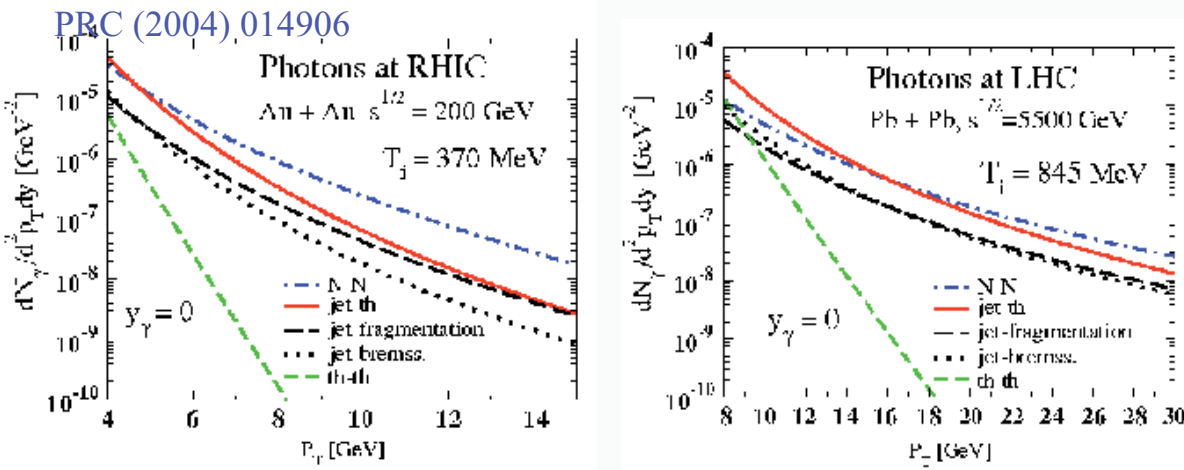

Photons are abundantly produced at LHC

Jet-photon conversion in the plasma dominates $8<p_{T}<14 \mathrm{GeV}$

Primordial hard NN scattering dominant for $P_{T}>20 G e V$ at $L H C$ 


\section{How can we distinguish different photon sources?}

- Prompt : $R_{A A}=1, v_{2}=0$ (azimuthal anisotropy)

- Fragmentation: $R_{A A}<1, v_{2}>0$

- Thermal, Bremsstrahlung, Jet Conversion: $R_{A A}>1, v_{2}<0$

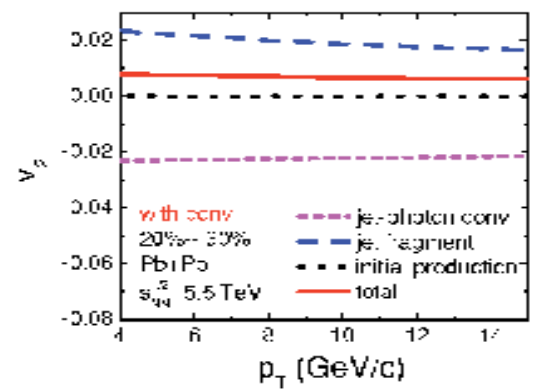

$R_{A A}>1$ and $v_{2}<0$

Unambiguous signature of medium produced photons

W.Liu, R.J.Fries nu-th/0801.0453

\section{Idlentiffying prompt $\gamma$ in ALICE}

\section{Isolation method}

- $\mathrm{pp}$

- $R=0.3, \Sigma p_{\mathrm{T}}<2 \mathrm{GeV} / \mathrm{c}$

- Efficiency: 69\%

- Background rejection: 1/170

- First year (10 $\left.\mathrm{pb}^{-1}\right)$

$$
\text { - } 3000 \gamma(E \gamma>20 \mathrm{GeV})
$$

Y. Mao, Poster QM2008,

ALICE-INT-2007-021

- $\mathrm{PbPb}$

- $R=0.2, p_{\mathrm{T}}^{\text {thresh }}=2 \mathrm{GeV} / \mathrm{c}$

- Efficiency: $50 \%$

- Background rejection: 1/14

- One month of running

- $2000 \gamma\left(E_{\gamma}>20 \mathrm{GeV}\right)$

G. Conesa et al., NIM A 580(2007) 1446, NIM A 585(2008) 28
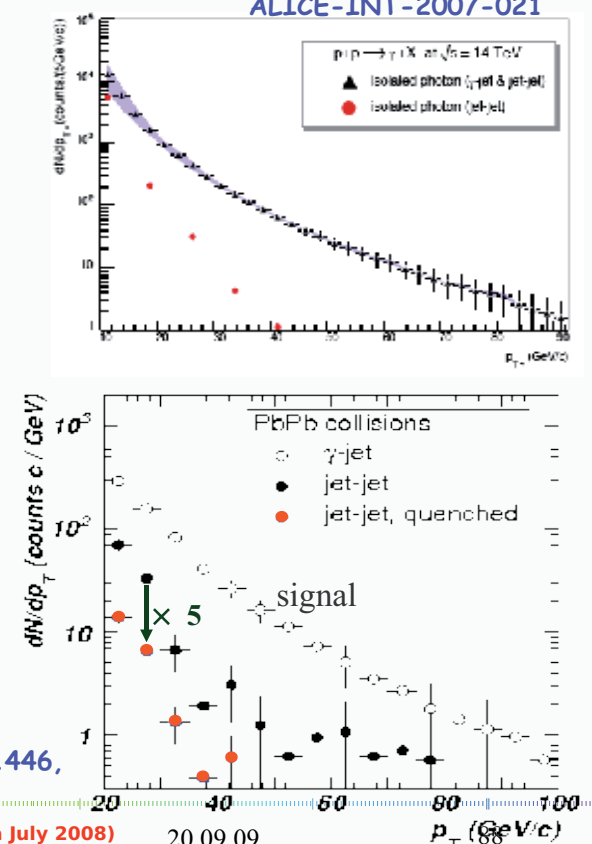


\section{Direct photons}

\section{Sensitivity of subtraction method}

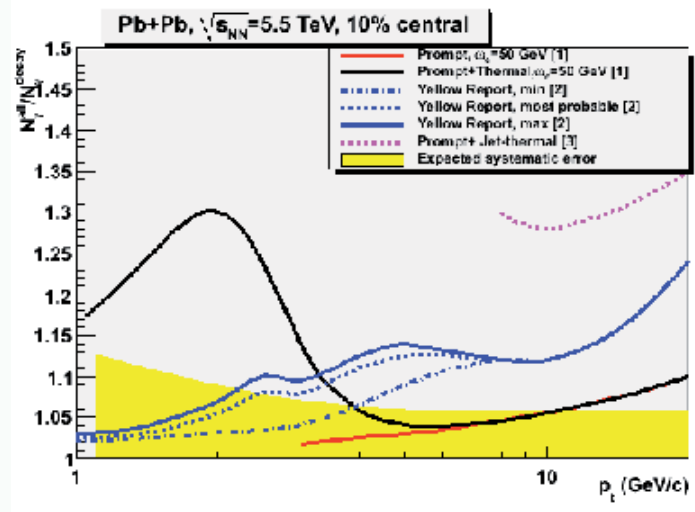

D. Peressounko, Hard Probes 2008
[1] N. Arimesto: (ed.i et al. F.Arleo et \&l.

J.Phys.G35:054001, 2008

$2+1$ lydio: $T_{11} 650$ MeV, reect: $7 \mathrm{HG} \equiv \mathrm{OS}$

POCD: CTEO5.5 M + nDSg: AKK-m:=50 GoV

[2] F. Arleo et al , Yetow Reporthep-phi0311131

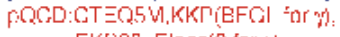

EKS9g-Eloss? for '

[3] S.Tukide el al., Phys.Rev.C72:014906,2005

Upper $p_{t}$ limit is defined by

- Available statistics

- Ability to separate $\pi^{0}$ and single photons $\sim 50 \mathrm{GeV}$

\section{Jets and Jet quenching (l)}

- Jets : reflect interactions of partons in partonic matter -Effects

-Reduction of single inclusive high pt particles

-Parton specific (stronger for gluons than quarks)

-Flavour specific (stronger for light quarks)

-Measure identified hadrons ( $p, K, p, \Lambda$, etc.) + heavy partons (charm, beauty) at high $p T$

- Change of fragmentation function for hard jets ( $p t>10 \mathrm{GeV} / \mathrm{c}$ )

-Transverse and longitudinal fragmentation function of jets

-Jet broadening $\rightarrow$ reduction of jet energy, dijets, 9 -jet pairs 


\section{Jets and Jet Quenching (II)}

- Experimental Consequences

- Measurement of Jet Energy is important

- Initially ALICE measures only charged particles and electromagnetic energy in PHOS

- Large EM Calorimeter under construction and will provide significant performance bonus

- Measurement of Jet Structure very important

- Requires good momentum analysis from 1Gev/c to 100 Gev/c

- ALICE excels in this domain

- pp and PA measurements essential as reference for physics in cold nuclear matter

\section{Jet quenching}

- Excellent jet reconstruction... but challenging to measure global medium modification ...

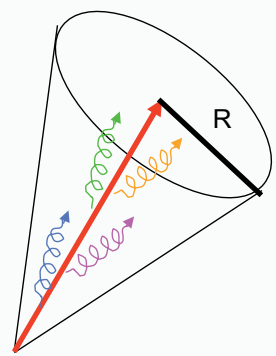

Medium induced redistribution of jet energy occurs inside cone

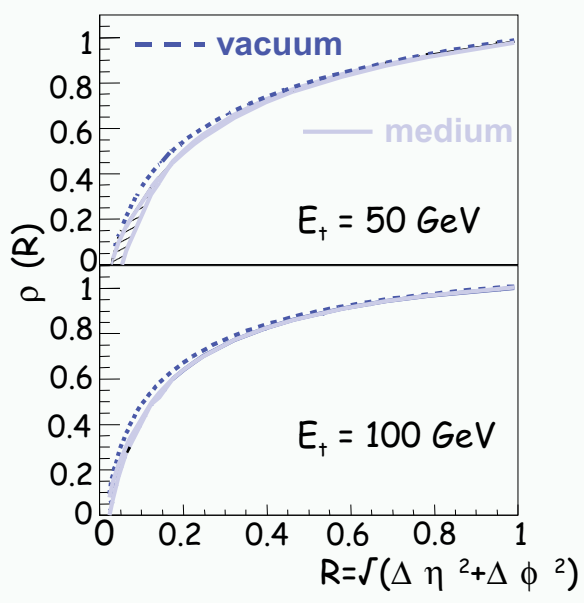

- $\quad E_{t}=100 \mathrm{GeV}$ (reduced average jet energy fraction inside $\mathbf{R}$ ):

- Radiated energy $\sim 20 \%$

- $R=0.3$ : $D E / E=3 \%$ 


\title{
Why photon-tagged jets?
}

- Medium effects redistribute $\left(\propto q_{l}^{\wedge}\right)$ the parton energy, $E_{j t t}$ inside the hadron jet (multiplicity, $j_{T}$ ).

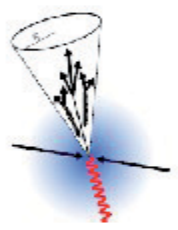

- Redistribution can be best measured with the Fragmentation Function... If we know $E_{j \mathrm{jt}}$

- HI environment hinders precise reconstruction of $E_{\text {jet. }}$

If we measure $\mathrm{E}_{\gamma} \approx$ $E_{\text {jet }}$

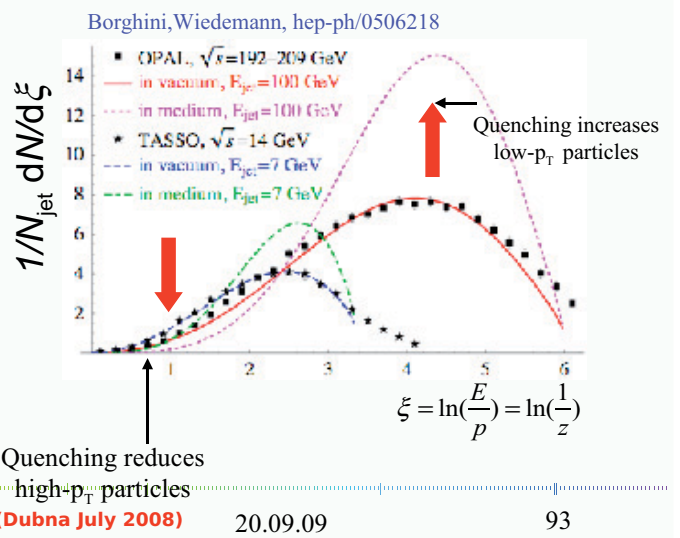

\section{Full Simulation and Reconstruction Jet Spectra}

\author{
Reconstruction efficiency dominated \\ by smearing of the spectrum \\ Charged jets: $33 \%$ \\ Charged + neutral: $68 \%$
}
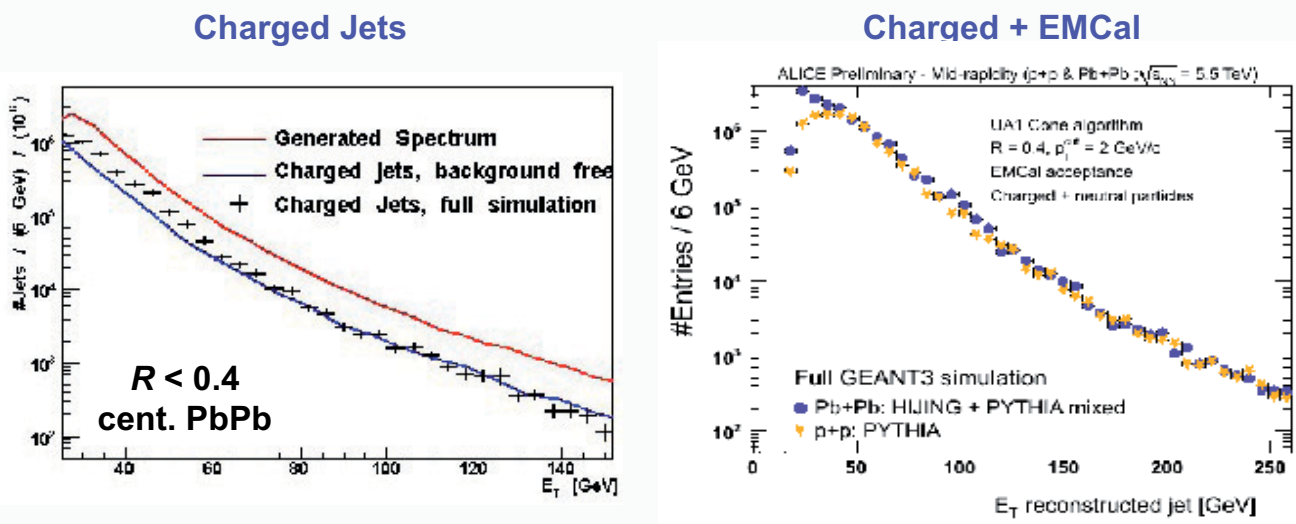

Statistics for 1 month of $\mathrm{PbPb}$ running 


\section{Jet Production at LHC}

- Initial measurements up to $100 \mathrm{GeV}$ (untriggered charged jets only)

- Detailed study of fragmentation possible

- Sensitive to energy loss mechanism

- Accuracy on transport coefficient < q > 20\%

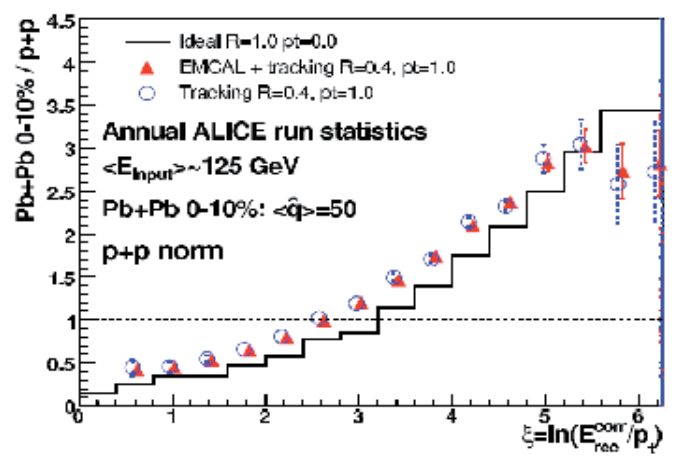

\begin{tabular}{|c|c|c|}
\hline $\begin{array}{c}\mathrm{p}_{\mathrm{t}} \text { jet }> \\
(\mathrm{GeV} / \mathrm{c})\end{array}$ & $\begin{array}{c}\text { jets/event } \\
\mathrm{Pb}+\mathrm{Pb}\end{array}$ & $\begin{array}{c}\text { accepted jets/ } \\
\text { month }\end{array}$ \\
\hline 5 & $3.510^{2}$ & $4.910^{10}$ \\
\hline 50 & $7.710^{-2}$ & $1.510^{7}$ \\
\hline 100 & $3.510^{-3}$ & $8.110^{5}$ \\
\hline 150 & $4.810^{-4}$ & $1.210^{5}$ \\
\hline 200 & $1.110^{-4}$ & $2.810^{4}$ \\
\hline
\end{tabular}

ense Matter in Heavy Ion Collisions and Astrophysics, A. Marin (Dubna July 2008)

20.09.09

\section{ALICE: Photon-Tag Jets Fragmentation Function}

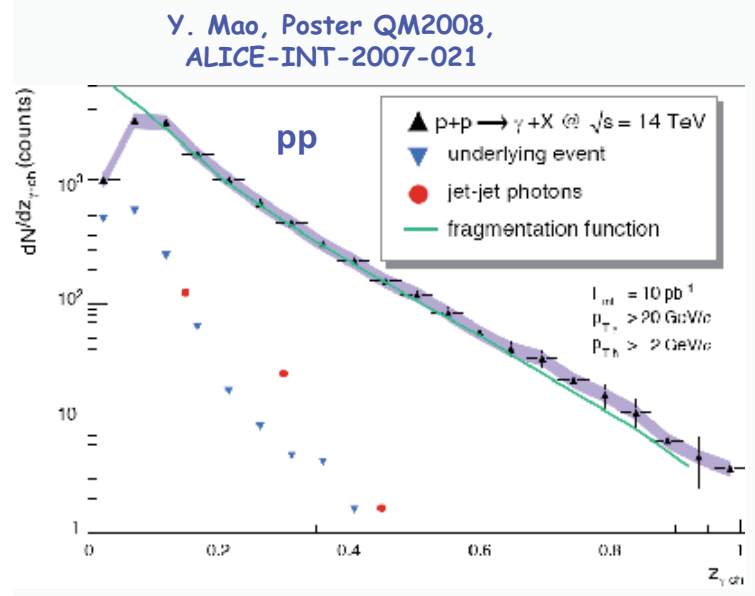

Imbalance distribution
G. Conesa et al., NIM A 580(2007) 1446,

NIM A 585(2008) 28

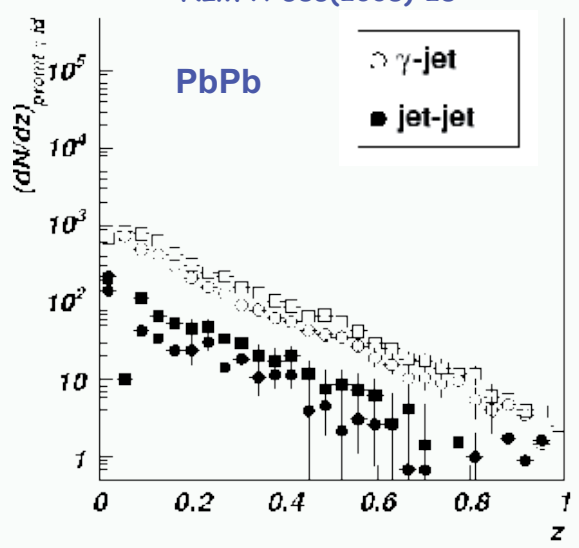

Isolated photons and background subtracted 


\section{ALICE: $\gamma$-tagged FF $R_{A A}$}

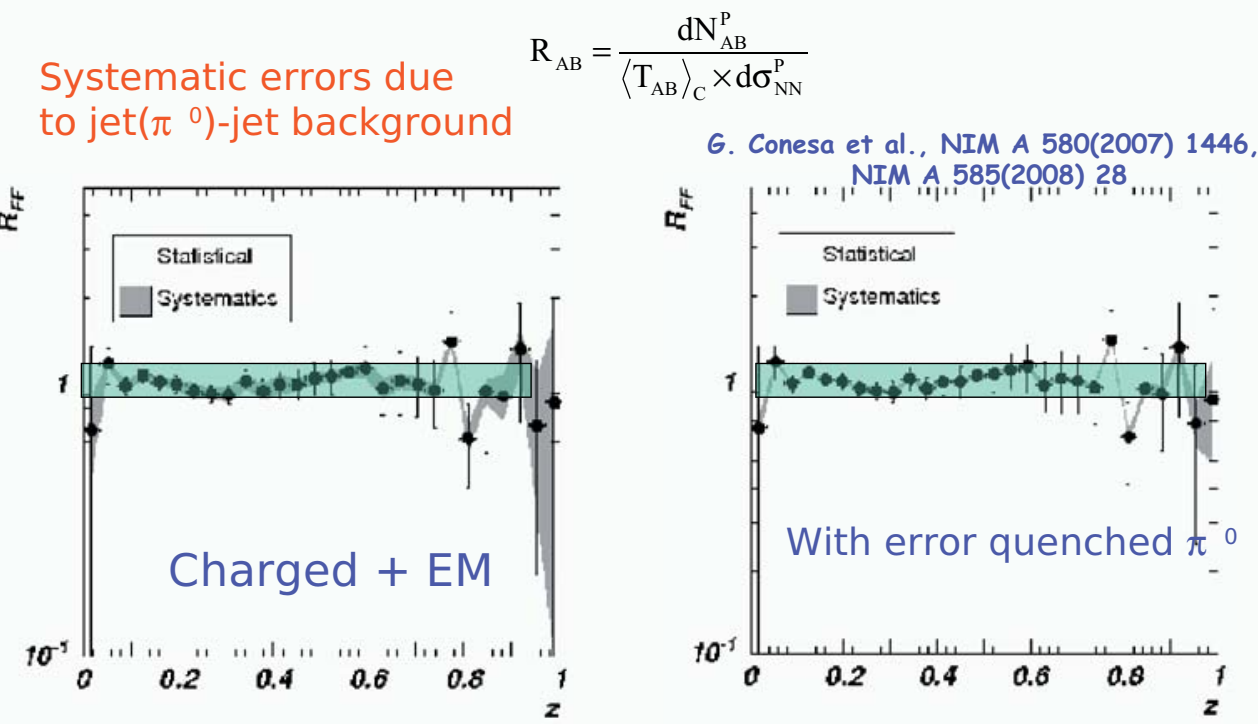

Sensitive to medium modifications at low $z$

\section{Bibliography}

- ALICE PPR Vol I: CERN/LHCC 2003-049; ALICE coll. J. Phys. G30, 1517 (2004)

- ALICE Physics - theoretical overview

- $L H C$ experimental conditions

- ALICE detector

- Offline computing and Monte Carlo generators

- ALICE PPR Vol II: CERN/LHCC 2005-030 (part 1 - part 2); ALICE coll. J. Phys. G32, 1295 (2006)

- Detector performance

- ALICE physics performance

- http://aliceinfo.cern.ch

- QCD and Collider Physics. R.K. Ellis, W.J. Stirling and B.R. Webber

- Ultrarelativistic Heavy-Ion Collisions. Ramona Vogt. 


\section{Bibliography}

- "The large hadron collider". Nature, Vol 448, 19 July 2007.

- Review of Particle Physics, W.-M. et al., Journal of Physics G33 (2006) 1.

\section{THE END}

\section{http://www.lhcountdown.com/}

\section{Thanks to the ALICE collaborators}

